\title{
Exact Solutions of Planar Photonic Crystal Waveguides with Infinite Claddings
}

\author{
By \\ Soheilla Mirlohi

\begin{abstract}
Thesis submitted to the Faculty of the
Virginia Polytechnic Institute and State University

in partial fulfillment of the requirements for the degree of

Master of Science

in

Electrical Engineering
\end{abstract}

Dr. Ahmad Safaai-Jazi , Chairman

Dr. Ioannis M. Besieris

Dr. David L. Russell

September, 2003

Blacksburg, VA

Keywords: Photonic Crystal Waveguides, Planar Optical waveguides, Waveguides with Periodic Structures

Copyright 2003, Soheilla Mirlohi 


\title{
Exact Solutions of Planar Photonic Crystal Waveguides with Infinite Claddings
}

\author{
By \\ Soheilla Mirlohi \\ Committee Chairman: Dr. Ahmad Safaai-Jazi
}

\begin{abstract}
A theoretical investigation of one-dimensional planar photonic crystal waveguides is carried out. These waveguides consist of a dielectric layer sandwiched between two semiinfinite periodic dielectric structures. Using a novel approach, exact analytical solutions for guided modes in such waveguides are presented. The se rigorous solutions allow one to distinguish clearly between the index-guiding regime and guidance due to the photonic crystal effect.
\end{abstract}

In the first part of this investigation, a rigorous analysis of the reflection of uniform plane waves from a semi-infinite periodic dielectric structure is undertaken. Both parallel and perpendicular polarizations for the incident plane wave are considered. Exact expressions for the reflection coefficients corresponding to two polarization cases are obtained using an impedance approach.

The results for the reflection coefficient are then used to study propagation properties of guided modes in one-dimensional photonic crystal waveguides with semiinfinite periodic cladding regions. Characteristic equations, from which propagation constants of guided modes can be obtained, and solutions for electromagnetic fields of these modes are derived. Solutions for both TE (transverse electric) and TM (transverse magnetic) modes are presented. Numerical results for the propagation constant and field distributions of several lower-order modes are presented. The solutions unique to photonic crystal waveguides are emphasized. 


\section{Acknowledgments}

First of all I like to give a special thanks to my advisor Dr. Ahmad Safaai-Jazi for his continuous and invaluable guidance. I shall always remember him for his kindness and all the support that he has given me throughout my studies. I have been so fortunate and honored for the opportunity that I have been given to learn from many great educators such as Dr. Safaai-Jazi, and the members of my committee, Dr. Ioannis Besieris and Dr. David Russell. I am forever grateful to them.

I would also like to thank my father, brothers, and sister for their never-ending supports. At the end, I wish to dedicate this work to my Mother. I hope she can look down from above and know that it has been only her encouragements and her beautiful smile that got me to where I am today, and makes me keep on going. 


\section{Table of Contents}

$\begin{array}{lll}\text { Chapter 1. Introduction } & 1\end{array}$

1.1 Background 1

1.2 Photonic Crystal waveguides 2

1.3 Scope of investigation 6

Chapter 2. Reflection and refraction from a semi-infinite periodic structure $\quad 8$

2.1 Reflection from a dielectric slab 9

2.2 Reflection from a semi-infinite periodic dielectric structure 11

Chapter 3. Analysis of Planar 1-D Photonic Crystal Waveguide with Infinite $\begin{array}{ll}\text { Cladding } & 17\end{array}$

3.1 Characteristic Equations 17

3.2 Slab Waveguide as Special Case 21

3.3 Field Analysis 22

3.3.1 TE Modes 23

3.3.2 Boundary Conditions 26

3.3.3 TM Modes 28

3.3.4 Fields Solutions Corresponding to Photonic Crystal Effect 31

3.4 Waveguide with Finite Number of Cladding Layers 32

Chapter 4. Numerical Results $\quad 34$

4.1 Wave Impedance 34

4.2 Propagation Characteristics of One-Dimensional Planar Photonic Crystal Waveguides 40

$\begin{array}{ll}4.3 \text { Field distributions } & 44\end{array}$

Chapter 5. Conclusions and Suggestions for Further Research 50

5.1 Conclusions $\quad 50$

5.2 Suggestions for Further Research $\quad 50$

$\begin{array}{ll}\text { References } & 52\end{array}$ 


\section{List of Figures}

Figure 1.1 Photonic crystals. (a) 1D, (b) 2D, and (c) 3D periodicity. 1

Figure. 1.2. A schematic view of one-dimensional photonic crystal dielectric structure. 3

Figure 2.1 Index profile of one-dimensional planar photonic crystal waveguide 8

Figure 2.2 Reflection and refraction of plane wave by a dielectric slab of thickness d 9

Figure 2.3 Reflection from a semi-infinite periodic dielectric planar structure 11

Figure 2.4 Equivalent structure for calculation of input impedance of semi-infinite $\begin{array}{ll}\text { periodic structure. } & 12\end{array}$

Figure 3.1 Front view of 1D planar photonic crystal waveguide 18

Figure 4.1 Variations of the real part of normalized wave impedance, $\operatorname{Re}\left[\bar{Z}_{i n}\right]$, versus incident angle $\boldsymbol{\theta}_{i}$ for the case of '-' solution and parallel polarization at the wavelengths (a) $\lambda=0.5 \mu \mathrm{m}$, (b) $\lambda=1.0 \mu \mathrm{m}$, (c) $\lambda=1.5 \mu \mathrm{m}$, (d) $\lambda=2.0 \mu \mathrm{m}$. The parameters of the semi-infinite structure are $n_{1}=1.5, n_{2}=1, d_{1}=1.5 \mu \mathrm{m}$, and $d_{2}=1.0$ $\mu m$.

Figure 4.2 Variations of the imaginary part of normalized wave impedance, $\operatorname{Im}\left[\bar{Z}_{\text {in }}\right]$, versus incident angle $\theta_{i}$ for the case of '-' solution and parallel polarization at the wavelengths (a) $\lambda=0.5 \mu \mathrm{m}$, (b) $\lambda=1.0 \mu \mathrm{m}$, (c) $\lambda=1.5 \mu \mathrm{m}$, (d) $\lambda=2.0 \mu \mathrm{m}$. The parameters of the structure are the same as those in Figure 4.1.

Figure 4.3 Variations of the real part of normalized wave impedance, $\operatorname{Re}\left[\bar{Z}_{i n}\right]$, versus incident angle $\theta_{i}$ for the case of '-' solution and perpendicular polarization at the wavelengths (a) $\lambda=0.5 \mu \mathrm{m}$, (b) $\lambda=1.0 \mu \mathrm{m}$, (c) $\lambda=1.5 \mu \mathrm{m}$, (d) $\lambda=2.0 \mu \mathrm{m}$. The parameters of the structure are the same as those in Figure 4.1.

Figure 4.4 Variations of the imaginary part of normalized wave impedance, $\operatorname{Im}\left[\bar{Z}_{\text {in }}\right]$, versus incident angle $\theta_{i}$ for the case of '-' solution and perpendicular polarization at the wavelengths (a) $\lambda=0.5 \mu \mathrm{m}$, (b) $\lambda=1.0 \mu \mathrm{m}$, (c) $\lambda=1.5 \mu \mathrm{m}$, (d) $\lambda=2.0 \mu \mathrm{m}$. The parameters of the structure are the same as those in Figure 4.1.

Figure 4.5 Comparison of the normalized propagation constant of $\mathrm{TE}_{2}$ mode at $\lambda=1.8$ $\mu \mathrm{m}$ for waveguides with finite and infinite cladding layers. 
Figure 4.6 Variations of normalized propagation constant versus wavelength for several lower-order modes in a planar photonic crystal waveguide with parameters $a$ $=1 \mu m, d_{1}=1 \mu m, d_{2}=1 \mu m, n_{1}=1.5$, and $n_{2}=1$.

Figure 4.7 Variations of normalized propagation constant versus wavelength for modes supported due to the photonic crystal effect. Waveguide parameters are the same as those in Figure 4.6.

Figure 4.8 Field distributions for the first even TE mode $\left(\mathrm{TE}_{0}\right)$ at $\lambda=1.5 \mu m$. The waveguide parameters are the same as those in Figure 4.6. Solid line for $h_{z}$ multiplied by 1000, dashed line for $e_{y}$, and dashdot line for $h_{x}$ multiplied by 1000 .

Figure 4.9 Field distributions for the first odd TE mode $\left(\mathrm{TE}_{1}\right)$ at $\lambda=1.5 \mu \mathrm{m}$. The waveguide parameters are the same as those in Figure 4.6. Solid line for $h_{z}$ multiplied by 1000, dashed line for $e_{y}$, and dashdot line for $h_{x}$ multiplied by 1000 .

Figure 4.10 Field distributions for the second even TE mode $\left(\mathrm{TE}_{2}\right)$ at $\lambda=1.5 \mu m$. The waveguide parameters are the same as those in Figure 4.6. Solid line for $h_{z}$ multiplied by 1000, dashed line for $e_{y}$, and dashdot line for $h_{x}$ multiplied by 1000 .

Figure 4.11 Field distributions for the second odd TE mode $\left(\mathrm{TE}_{3}\right)$ at a) $\lambda=1 \mu \mathrm{m}$ for $\bar{\beta}>\mathrm{n}_{2}$, and b) $\lambda=1.5 \mu m$ for $\bar{\beta}<\mathrm{n}_{2}$. The waveguide parameters are the same as those in Figure 4.6. Solid line for $h_{z}$ multiplied by 1000, dashed line for $e_{y}$, and dashdot line for $h_{x}$ multiplied by 1000 . 


\section{Chapter 1. Introduction}

\subsection{Background}

Photonic crystal waveguides have been the subject of extensive research in recent years. This chapter presents some background information about different types of fiber, as well as planar photonic crystal waveguides. In particular, the one-dimensional planar photonic crystal waveguides, which are the focus of our investigation, are discussed in more details. The idea of "photonic crystals" started from the notion of Bragg condition in periodic structures under which light at particular wavelengths completely reflects off such structures. As shown in Figure 1.1, there are three different types of periodic structures; namely, one-dimensional (1D), two-dimensional (2D), and three-dimensional (3D) periodic structures [1].

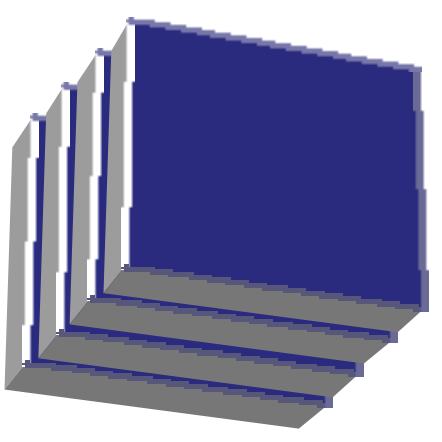

(a)

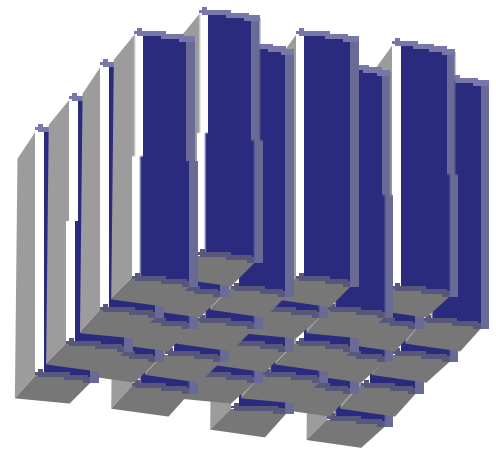

(b)

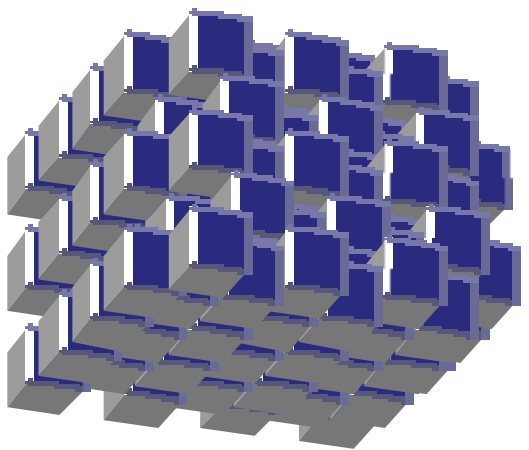

(c)

Figure 1.1 Photonic crystals. (a) 1D, (b) 2D, and (c) 3D periodicity.

It is this periodicity in the refractive index that gives rise to the condition under which certain wavelengths can pass through, while a select wavelength is reflected. These frequency gaps, where the propagation of electromagnetic waves is forbidden, are defined as 'photonic band gaps.' One dimensional crystals are periodic in only one axial direction, such as a stack of alternating refractive-index planar dielectric layers as in Fig. 1a, whereas 2D-periodic photonic crystals are periodic along two directions, and 3D- 
periodic photonic crystals are periodic in all three axial directions. The main advantage of $3 \mathrm{D}$ over $2 \mathrm{D}$ periodic structures is their flexibility to channel light in any direction. This means, regardless of the type of polarization or the direction of propagation, light in certain band of frequencies cannot propagate in 3D photonic crystals [2]. But since the manufacturing of 3D photonic crystals is complex and more costly, 2D-periodic photonic crystals are the type more widely used. Due to their ability to confine light effectively, the planar photonic crystals can facilitate the creation of all-photonic integrated circuits (ICs). Another example of the use of 2D photonic crystal structures is the photonic crystal fiber, also known as holey fiber. Some of the functionalities supported by various designs of photonic crystal fibers include insensitivity to bending, reduced absorption loss, zero dispersion, polarization stability, and high-power delivery. The best characteristics of photonic crystals are that they can reflect, trap, and guide light at particular wavelengths. The following section presents a more detailed description of different types of photonic crystal waveguides and their properties, which are currently under investigation.

\subsection{Photonic Crystal waveguides}

The exciting point in using photonic crystals is the possibility of designing waveguides with such materials that could trap or prohibit the propagation of light at any wavelength; not only microwaves, but also infrared waves and visible light waves. The underlying principle behind prohibition of propagation of light (or electromagnetic waves in general) along a certain direction in a periodic dielectric structure is the destructive interference of reflected and refracted components of light in a particular frequency range. The smaller the wavelength, the smaller the periodicity in crystal structure has to be [3]. One type of photonic crystal waveguide with a wide range of applications is the photonic crystal fiber (PCF). Compared to conventional optical fibers, where the core material is different from that of the cladding, the PCF is made up of a single-material. In PCFs, a core of pure silica is embedded within a periodic array of air holes which form a cladding [4]. The light in conventional optical fiber is guided and confined to a central core by total internal reflection from the core-cladding boundary. In photonic crystal 
fibers, the guidance of light is maintained not only due to total internal reflection, as the region with air holes behaves like a cladding with an average refractive index lower than the index of the solid core, but also due to the photonic band gap effect. The latter effect can contribute more significantly to light guidance in hollow-core holey fibers [5]. The positioning and size of the holes affect the way light is transmitted by the fiber and allows different properties, such as nearly zero or high chromatic dispersion and highly nonlinear or linear behavior, to be achieved [6-8]. Another unique property of holey fibers are that they stay single-mode over a wide range of wavelengths [9].

The research presented in this thesis focuses on planar one-dimensional photonic band gap structures. Compared to 2D and 3D structures, the one-dimensional photonic band gap structures are easier to fabricate and lend themselves to rigorous analytical solutions. The 1D planar photonic band gap structures find applications in distributed feedback lasers, optical filters, and wavelength selective micro-optic devices. A 1D planar photonic crystal structure consists of alternating layers of material with different dielectric constants as shown in Figure 1.2. It is because of this contrast between the dielectric constants of two neighboring layers that photonic band gaps exist.

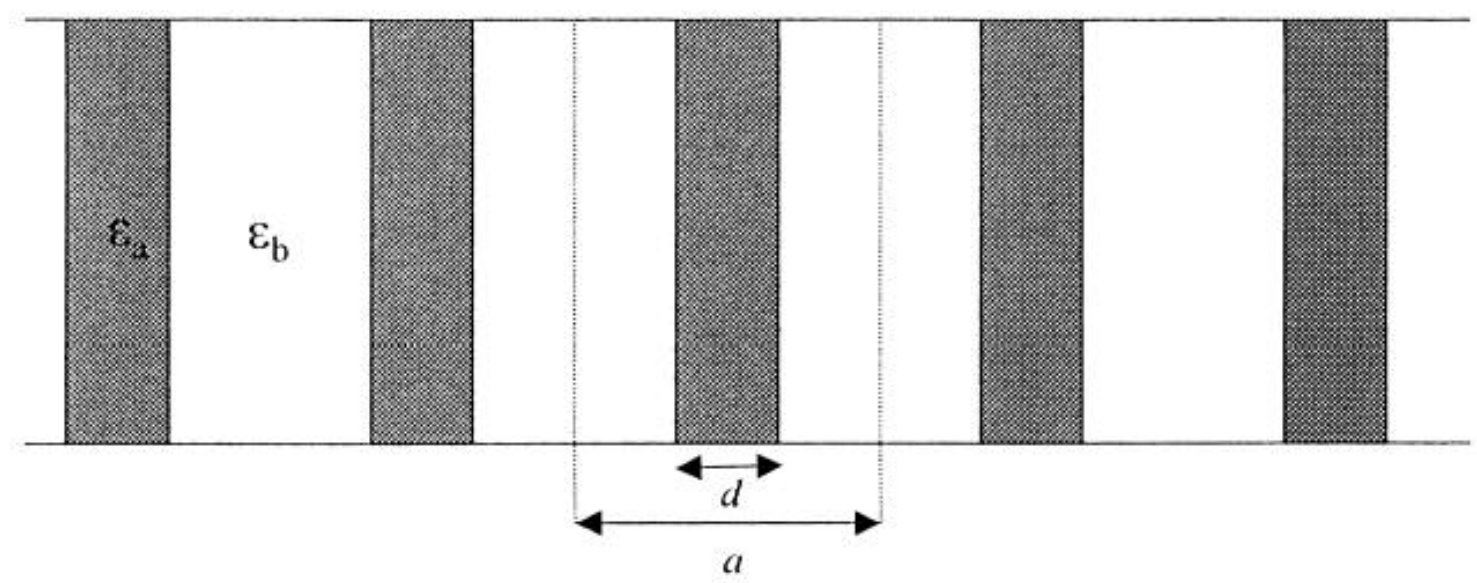

Figure. 1.2. A schematic view of one-dimensional photonic crystal dielectric structure.

It has been shown that the larger the difference between the two indices the wider the band gaps become. Also, as the width of the air layers become smaller in comparison to the width of the dielectric layers, the width of band gaps would decrease. The angle of 
incidence of the light wave is also another factor which effects the width of band gaps [3].

The concept of photonic band gaps in crystals also exists in other materials such as metals, liquid crystals, and polymers. One of these materials, called block copolymer, is made of chemically different macromolecules or blocks, which can be made into one-, two-, and three-dimensional periodic dielectric structures. Each layer is formed by a different block. Although, small band gaps are formed because of the small contrast between dielectric constants of layers, it is possible to create band gaps in the visible light spectrum. There are different methods which can be used to increase this contrast. In their two and three-dimensional forms, they are able to produce complete photonic band gaps of both TE and TM polarizations. Such factors as reflection of certain frequencies in the gap are dependent on the index contrast and the number of periodic layers; the lower the contrast is, the higher the number of layers must be [10].

Another type of band gap structure is a sandwich configuration in which a periodic pattern of square metal pads is printed in the midmost of a substrate. This design can be fabricated through printed circuit board technology. The periodic lattice is printed on the upper face of a board, while another board is placed on top with the same microstrip line of periodic structure. The two lines are placed on top of each other, creating a periodic structure right in the middle of substrate. The band gap width can be adjusted by simply moving the vertical location of the periodic strip line. This structure eliminates the need for drilling holes, and it can be applied to circuit design to simplify its structure and decrease the dimensions [11].

Photonic crystals may also be used to make devices for wavelength division multiplexing (WDM). An example of this is a one-dimensional dielectric stack structure, made of a numbers of periodic layers of two materials with different dielectric constants. When a light beam is incident at an angle, a certain range of wavelengths is reflected because of photonic band gap in the structure. For those wavelengths, which are close to the photonic band edge, the position of the beam shifts by a few micro meters; therefore, a beam of different wavelengths can be separated. When the thickness of the stack 
structure or the number of periods is increased, the separation of the beams becomes larger; up to a point where the separation stays constants and the number of layers in the structure is considered to be approximately "infinite" [12].

One particularly interesting aspect of photonic crystals is the possibility of creating crystal defects that confine light in localized modes; the creation of these modes are highly sensitive to the structure of defects [13,14]. Although in the band gap frequency range electromagnetic waves cannot propagate or are totally reflected, a band of wavelengths appears within the photonic band gap to be guided when the periodicity of crystal structure is broken or a defect is introduced into it [15]. Beside the refractive index in photonic crystals, the wave impedance also has a role in the appearance of photonic band gaps $[16,17]$. The waveguiding is possible through two types of defects, the line defect and the point defect. As an example, $\mathrm{b}$ manipulate the waveguiding properties of a slab, researchers have created a range of thin silicon slabs with 2D lattice holes that contain a row of missing holes or line defect. By altering the width of the line defect, we are able to change the 'wavelength window' within which light propagates through the line defect waveguide. The mode properties depend strongly on the line defect width. By removing a single line of holes, the simplest photonic crystal waveguide is formed. In such a structure, light is confined to the waveguide in the vertical direction by the means of total internal reflection, and in the lateral direction by distributed Bragg reflection, due to the presence of 2D photonic crystal [18].

In conventional index-guided waveguides, optical losses are large around sharp bends. For this reason, photonic crystals can be used for guiding light around corners, with near perfect transmission. Another example of a photonic crystal waveguide is a $2 \mathrm{D}$ periodic array of cylindrical rods of high-index dielectric material embedded on a low-index dielectric material. A line defect is introduced by reducing the radius of a row of cylinders. The periodic arrangement of dielectric rods surrounding the line defect creates a photonic band gap. Thus, an optical signal with frequency within the photonic band gap has its energy confined in the line defect, provided that the line defect is sufficiently wide to support a well confined guided mode. Modes lying within the band gap 
wavelength range are expected to follow a sharp bend in the defect waveguide, with little or zero loss due to back-reflectance from the corner points. This facilitates the fabrication of tight waveguide bends for the fabrication of smallscale waveguide splitters and combiners [19,20]. Hence, it is the localization of a mode inside the line defect that can guide light around sharp corners including a 90' bend with low optical loss. This is useful in large-scale photonic integrated circuits, where guiding light around sharp bends is required [21]. It is through the use of defects that the design of optical components such as filters and integrated optical circuits becomes possible.

Although defects can be created by either adding or removing high-index materials, it is the reduced-index case that is unique to photonic crystals. Traditional dielectric waveguides, which operate on the principle of index guiding, necessarily confine light to regions of higher index. In contrast, photonic-crystal waveguides can guide light in regions of lower index, even in air. In this case, a reduced-index line defect is created by increasing the size of one line of holes in a layer. Because of this structure, the states created inside the band gap will stay confined to the region of defect, creating a waveguide. In 3D photonic crystals, light confinement can be achieved in all three directions. This is possible by creating a point defect in the structure, by removing or changing the size of only one air hole or a dielectric rod. A photonic crystal with a point defect is essentially a resonator.

\subsection{Scope of investigation}

Although most optical waveguides used in communication systems have a cylindrical shape, planar waveguides are nevertheless of great interest as they are useful in integrated optics, and their planar geometry simplifies the fabrication process as well as the analysis of their propagation properties. The purpose of this work is to present a theoretical investigation of one-dimensional planar photonic crystal waveguides. Using a novel approach, exact analytical solutions for guided modes in such waveguides will be presented. These rigorous solutions allow one to distinguish clearly between the index- 
guiding regime and guidance due to the photonic crystal effect. In this study, the propagation characteristics and modal field distributions in one-dimensional planar photonic crystal waveguides are considered. The structure of such waveguides is unique compared to a conventional slab waveguide in that the cladding regions consist of an infinite number of layers having a periodic refractive index profile.

Chapter 2 presents a rigorous analysis of the reflection of uniform plane waves from a semi-infinite periodic dielectric structure. Both parallel and perpendicular polarizations for the incident plane wave are considered. Exact expressions for the reflection coefficients corresponding to two polarization cases are obtained using an impedance approach. The results of chapter 2 are then used to study propagation properties of guided modes in one-dimensional photonic crystal waveguides with semiinfinite periodic cladding regions. Characteristic equations, from which propagation constants of guided modes can be obtained, and solutions for electromagnetic fields of these modes are in Chapter 3. Solutions for both TE (transverse electric) and TM (transverse magnetic) modes are presented. Chapter 4 presents the numerical results for example cases. In particular, dispersion characteristics and mode field distributions for several lower-order modes are presented. The solutions unique to photonic crystal waveguides will be emphasized. Finally, chapter 5 summarizes the conclusions of this research and points out suggestions for further research work. 


\section{Chapter 2. Reflection and refraction from a semi-infinite periodic structure}

The aim in this chapter is to investigate propagation properties of guided modes in a one-dimensional planar photonic crystal waveguide. Figure 2.1 shows the refractive index profile of this type of waveguide. Such a waveguide may be viewed as a dielectric region of thickness $2 \mathrm{a}$ and refractive index $\mathrm{n}_{1}$ sandwiched between two semi-infinite periodic structures (regions $x>a$ and $x<-a$ ). Thus, propagation of electromagnetic waves in this waveguide essentially amounts to multiple reflections between the two semiinfinite regions. With this view in mind, the analysis of reflection of waves incident on semi-infinite periodic structures would form a basis for studying the propagation of guided modes in one-dimensional planar photonic crystal waveguides. In this chapter, a novel impedance approach for the calculation of reflection coefficient for a plane wave incident at an arbitrary angle on a semi-infinite periodic structure is presented. The results of this analysis will then be used to obtain solutions for various guided modes supported by one-dimensional planar photonic crystal waveguides.

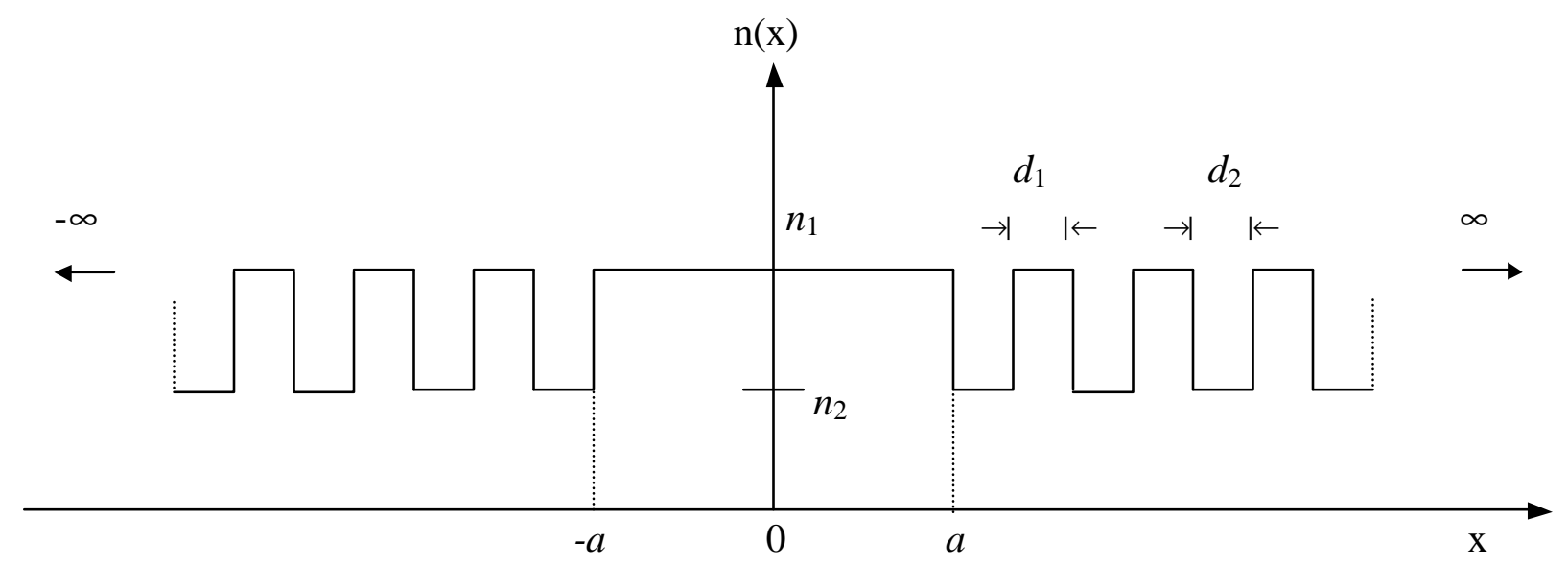

Figure 2.1 Index profile of one-dimensional planar photonic crystal waveguide 


\subsection{Reflection from a dielectric slab}

In order to examine reflection from a semi-infinite periodic structure, we first study reflection of a uniform plane-wave incident on a dielectric slab of thickness $d$. Two cases, corresponding to parallel ( $\|$ ) and perpendicular $(\perp)$ polarizations, are considered. These two cases are illustrated in Figure 2.2, where the incident, reflected, and transmitted waves together with the associated notations are shown. The reflection coefficient $\Gamma$, defined as $\Gamma=E^{r} / E^{i}$, can be obtained by writing the field expressions in the regions $\mathrm{x}<0$ (region of incident wave), $0<\mathrm{x}<\mathrm{d}$ (the dielectric slab region), and $\mathrm{x}>\mathrm{d}$ (the region of transmitted wave) and imposing the boundary conditions. The derivation of the reflection coefficients, although somewhat involved, is straightforward. The results are summarized as,

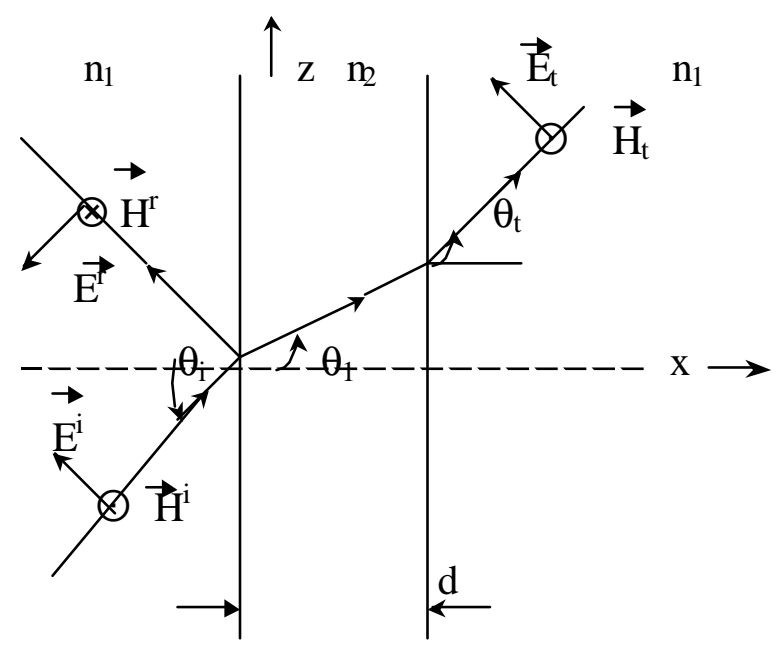

(a)

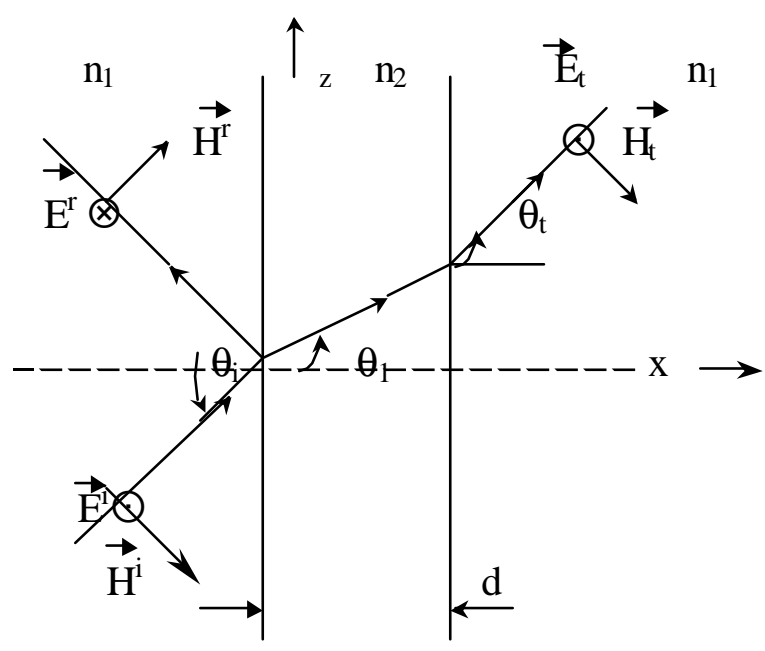

(b)

Figure 2.2 Reflection and refraction of plane wave by a dielectric slab of thickness d (a) Parallel polarization, (b) Perpendicular polarization. 


$$
\Gamma=\frac{\left(Z_{2}^{2}-Z_{1}^{2}\right)\left(1-e^{-j 2 \beta_{2} d}\right)}{\left(Z_{2}+Z_{1}\right)^{2}-\left(Z_{2}-Z_{1}\right)^{2} e^{-j 2 \beta_{2} d}}
$$

where $Z_{1}$ and $Z_{2}$ are wave impedances in regions $\mathrm{x}<0$ and $0<\mathrm{x}<\mathrm{d}$ as seen along the $\mathrm{x}$ axis; that is,

$$
\begin{aligned}
& Z_{1}=\left\{\begin{array}{l}
Z_{0} \cos \theta_{i} / n_{1}, \text { for } \| \text { polarization } \\
Z_{0} /\left(n_{1} \cos \theta_{i}\right), \text { for } \perp \text { polarization }
\end{array}\right. \\
& Z_{2}=\left\{\begin{array}{l}
Z_{0} \cos \theta_{1} / n_{2}, \text { for } \| \text { polarization } \\
Z_{0} /\left(n_{2} \cos \theta_{1}\right), \text { for } \perp \text { polarization }
\end{array}\right.
\end{aligned}
$$

and

$$
\begin{aligned}
& \beta_{2}=k_{0} n_{2} \cos \theta_{1}=k_{0} n_{2} \sqrt{1-\left(n_{1} \sin \theta_{i} / n_{2}\right)^{2}} \\
& Z_{0}=\sqrt{\mu_{0} / \varepsilon_{0}}=120 \pi \Omega \\
& k_{0}=\omega \sqrt{\mu_{0} \varepsilon_{0}}=\frac{2 \pi}{\lambda}
\end{aligned}
$$

with $\lambda$ being the free-space wavelength of the incident wave.

The reflection coefficient $\Gamma$ can be alternatively obtained using transmission line concepts and impedance transformation relationships. Using this approach, $\Gamma$ is obtained as follows:

$$
\Gamma=\frac{Z_{\text {in }}-Z_{1}}{Z_{\text {in }}+Z_{1}}
$$

where

$$
Z_{i n}=Z_{2} \frac{Z_{1}+j Z_{2} \tan \left(\beta_{2} d\right)}{Z_{2}+j Z_{1} \tan \left(\beta_{2} d\right)}
$$

In (2.7), the expressions for $Z_{1}, Z_{2}$, and $\beta_{2}$ are the same as those given in (2.2), (2.3), and (2.4), respectively. Upon substituting (2.8) in (2.7), it can be easily shown that (2.1) and (2.7) are identical. 


\subsection{Reflection from a semi-infinite periodic dielectric structure}

In the next step toward studying propagation properties of a waveguide with index profile as that of Figure 2.1, we examine reflection from a semi-infinite periodic dielectric structure. Figure 2.3 shows the geometry and parameters of such a structure. Reflection from an arbitrary but finite number of layers has been investigated before [22], but the extension to the case of infinite periodic layers is not, to our knowledge, available in the open literature. As discussed in the previous section, the impedance approach is used to calculate the reflection coefficient. In doing so, it should be noted that the input impedance seen at $\mathrm{x}=0$

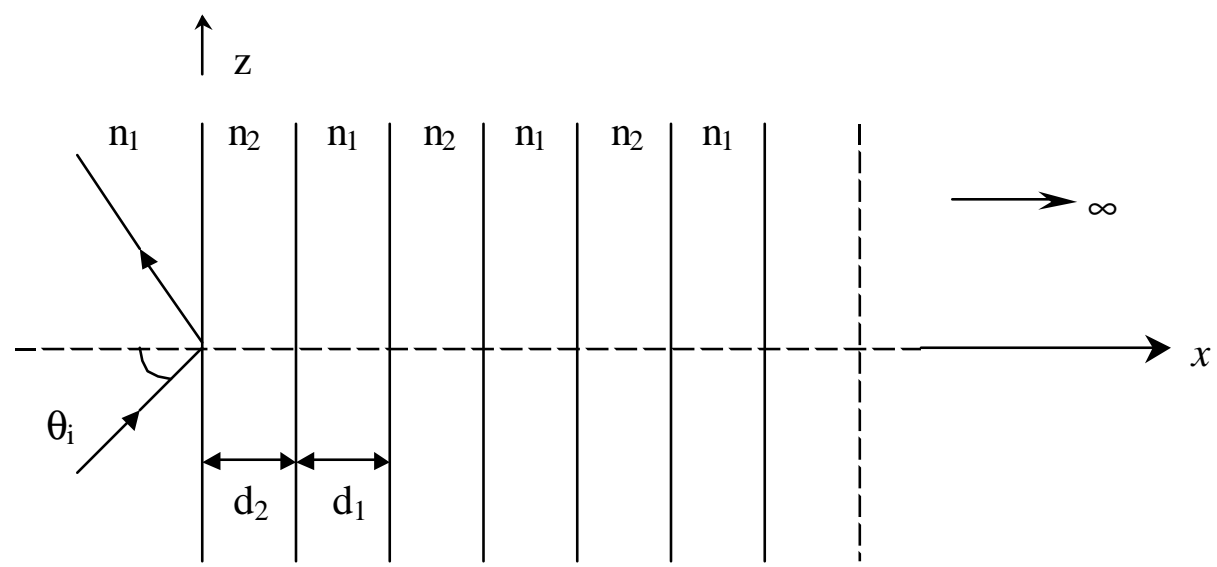

Figure 2.3 Reflection from a semi-infinite periodic dielectric planar structure

is the same as that seen at $\mathrm{x}=\mathrm{d}_{1}+\mathrm{d}_{2}$, because the wave sees the same structure looking along the $x$-axis. In other words, the region $x>d_{1}+d_{2}$ may be replaced by an equivalent medium with a wave impedance equal to $Z_{\text {in }}$ as depicted in Figure 2.4. This means, the wave impedance at the outer boundary $(\mathrm{x}=0)$ can be computed based on the impedance seen at the internal boundary $\left(\mathrm{x}=\mathrm{d}_{2}\right)$, which is defined as

$$
Z_{\text {in }}=Z_{\text {in }}(x=0)=Z_{2} \frac{Z_{\text {in }}^{\prime}+j Z_{2} \tan \left(\beta_{2} d_{2}\right)}{Z_{2}+j Z_{\text {in }}^{\prime} \tan \left(\beta_{2} d_{2}\right)},
$$

where 


$$
Z_{\text {in }}^{\prime}=Z_{\text {in }}\left(x=d_{2}\right)=Z_{1} \frac{Z_{\text {in }}+j Z_{1} \tan \left(\beta_{1} d_{1}\right)}{Z_{1}+j Z_{\text {in }} \tan \left(\beta_{1} d_{1}\right)}
$$

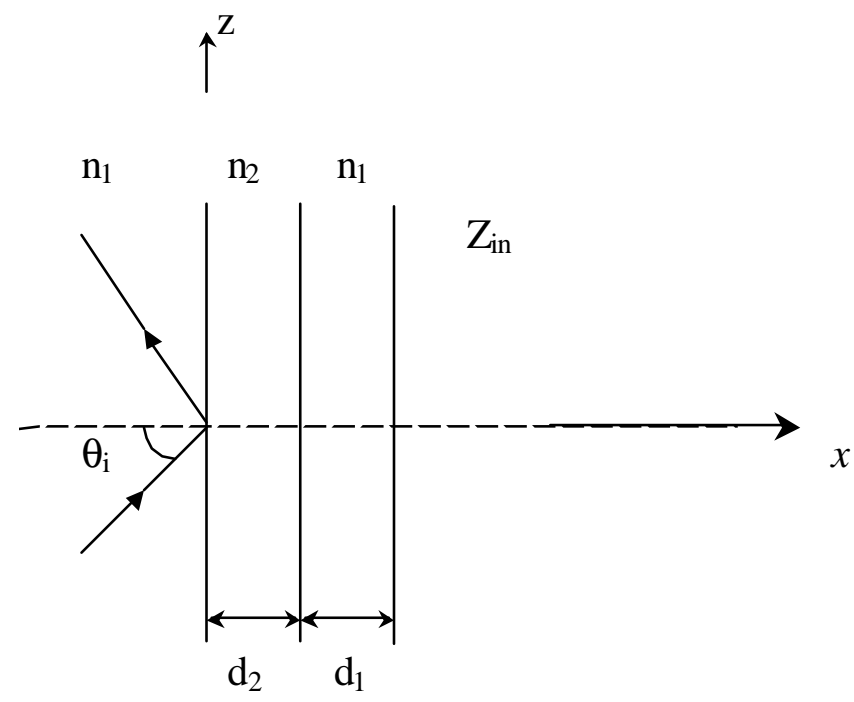

Figure 2.4 Equivalent structure for calculation of input impedance of semi-infinite periodic structure.

Substituting equation (2.10) in (2.9), yields

$$
Z_{\text {in }}=Z_{2} \frac{\frac{Z_{1}\left[Z_{i n}+j Z_{1} \tan \left(\beta_{1} d_{1}\right)\right]}{Z_{1}+j Z_{\text {in }} \tan \left(\beta_{1} d_{1}\right)}+j Z_{2} \tan \left(\beta_{2} d_{2}\right)}{Z_{2}+\frac{j Z_{1}\left[Z_{i n}+j Z_{1} \tan \left(\beta_{1} d_{1}\right)\right]}{Z_{1}+j Z_{i n} \tan \left(\beta_{1} d_{1}\right)} \tan \left(\beta_{2} d_{2}\right)}
$$

Simplifying and rearranging the terms in equation (2.11), we obtain

$$
a_{\mathrm{o}} Z_{i n}{ }^{2}+b_{\mathrm{o}} Z_{i n}+c_{\mathrm{o}}=0
$$

where 


$$
\begin{aligned}
& a_{\mathrm{o}}=Z_{1} \tan \left(\beta_{2} d_{2}\right)+Z_{2} \tan \left(\beta_{1} d_{1}\right) \\
& b_{\mathrm{o}}=j\left[\left(Z_{1}^{2}-Z_{2}^{2}\right) \tan \left(\beta_{1} d_{1}\right) \tan \left(\beta_{2} d_{2}\right)\right] \\
& c_{\mathrm{o}}=-Z_{1} Z_{2}\left[Z_{1} \tan \left(\beta_{1} d_{1}\right)+Z_{2} \tan \left(\beta_{2} d_{2}\right)\right]
\end{aligned}
$$

Equation (2.12) is quadratic in $\mathrm{Z}_{\mathrm{in}}$, which upon solving it yields,

$$
Z_{\text {in }}=\left(-b_{\mathrm{o}} \pm \sqrt{\Delta}\right) / 2 a_{\mathrm{o}}
$$

where $\Delta=b_{\mathrm{o}}{ }^{2}-4 a_{\mathrm{o}} c_{\mathrm{o}}$.

There are two solutions for $Z_{\text {in }}$ corresponding to + and - signs in equation (2.14). Clearly one of the solutions must be discarded. The answer should lie in the real part of $Z_{\text {in }}$; i.e., the resistive part of the input impedance, which must be positive. Further clarification will be provided later.

Let us now express $Z_{\text {in }}$ in a form more suitable for numerical computations. For the time being, let us assume that $\mathrm{n}_{1}>\mathrm{n}_{2}$. Then, for $\theta_{\mathrm{i}}>\theta_{\mathrm{c}}=\sin ^{-1}\left(\mathrm{n}_{2} / \mathrm{n}_{1}\right), \mathrm{Z}_{2}$ becomes purely imaginary because $\cos \boldsymbol{\theta}_{1}=\sqrt{1-\sin ^{2} \boldsymbol{\theta}_{1}}=\sqrt{1-\left(n_{1} \sin \boldsymbol{\theta}_{i} / n_{2}\right)^{2}}=j \sqrt{\left(n_{1} \sin \boldsymbol{\theta}_{i} / n_{2}\right)^{2}-1}$, as $\left(n_{1} \sin \theta_{i} / n_{2}\right)^{2}>1$. Introducing the notations,

$$
\begin{aligned}
& \bar{\beta}=\mathrm{n}_{1} \sin \theta_{\mathrm{i}} \\
& \mathrm{u}=\sqrt{\mathrm{n}_{1}{ }^{2}-\bar{\beta}^{2}} \\
& \mathrm{w}=\sqrt{\bar{\beta}^{2}-\mathrm{n}_{2}{ }^{2}}
\end{aligned}
$$

then

$$
Z_{1}=\left\{\begin{array}{l}
Z_{0} u / n_{1}^{2}, \text { for } \| \text { polarization } \\
Z_{0} / u, \text { for } \perp \text { polarization }
\end{array}\right.
$$




$$
Z_{2}=\left\{\begin{array}{l}
j Z_{0} w / n_{2}{ }^{2}, \text { for } \| \text { polarization } \\
-j Z_{0} / w, \text { for } \perp \text { polarization }
\end{array}\right.
$$

and

$$
\begin{array}{ll}
\beta_{1}=\mathrm{k}_{\mathrm{o}} \mathrm{n}_{1} \cos \theta_{\mathrm{i}}=\mathrm{k}_{\mathrm{o}} \mathrm{u}, & \beta_{1} d_{1}=k_{\mathrm{o}} u d_{1}=U, \\
\beta_{2}=\mathrm{k}_{\mathrm{o}} \mathrm{n}_{2} \cos \theta_{1}=\mathrm{jk} \mathrm{k}_{\mathrm{o}} \mathrm{w}, & \beta_{2} d_{2}=j k_{\mathrm{o}} w d_{2}=j W,
\end{array}
$$

substituting equations (2.17) to (2.19) in (2.13), we have

$$
\begin{aligned}
a_{0} & =Z_{0}\left[j \frac{u}{n_{1}^{2}} \tanh (W)+j \frac{w}{n_{2}^{2}} \tan (U)\right], \text { for } \| \text { polarization } \\
& =Z_{0}\left[j \frac{1}{u} \tanh (W)-j \frac{1}{w} \tan (U)\right], \text { for } \perp \text { polarization }
\end{aligned}
$$

where $\tan (\mathrm{jW})=\mathrm{jtanh}(\mathrm{W})$ has been used. For convenience $a_{0}$ is rewritten as

$$
a_{0}=j Z_{0} \bar{a}_{0}
$$

where

$$
\bar{a}_{0}=\left\{\begin{array}{l}
\left(\frac{u}{n_{1}^{2}}\right) \tanh (W)+\left(\frac{w}{n_{2}^{2}}\right) \tan (U), \text { for } \| \text { polarization } \\
\left(\frac{1}{u}\right) \tanh (W)-\left(\frac{1}{w}\right) \tan (U), \text { for } \perp \text { polarization }
\end{array}\right.
$$

In a similar manner, we have

$$
\begin{aligned}
& b_{0}=-Z_{0}{ }^{2} \overline{b_{0}} \\
& c_{0}=-j Z_{0}^{3} \bar{c}_{0}
\end{aligned}
$$

where 


$$
\begin{aligned}
& \bar{b}_{0}=\left\{\begin{array}{l}
{\left[\left(\frac{u}{n_{1}^{2}}\right)^{2}+\left(\frac{w}{n_{2}^{2}}\right)^{2}\right] \tan (U) \tanh (W), \text { for } \| \text { polarization }} \\
{\left[\left(\frac{1}{u}\right)^{2}+\left(\frac{1}{w}\right)^{2}\right] \tan (U) \tanh (W), \text { for } \perp \text { polarization }}
\end{array}\right. \\
& \bar{c}_{0}=\left\{\begin{array}{l}
\left(\frac{u w}{n_{1}^{2} n_{2}^{2}}\right)\left[\left(\frac{u}{n_{1}^{2}}\right) \tan (U)-\left(\frac{w}{n_{2}^{2}}\right) \tanh (W)\right], \text { for } \| \text { polarization } \\
-\left(\frac{1}{u w}\right)\left[\left(\frac{1}{u}\right) \tan (U)+\left(\frac{1}{w}\right) \tanh h(W)\right], \text { for } \perp \text { polarization }
\end{array}\right.
\end{aligned}
$$

Expressing $Z_{\text {in }}$ in terms of $\bar{a}_{0}, \bar{b}_{0}$, and $\bar{c}_{0}$ we obtain

$$
Z_{i n}=-j Z_{0}\left(\frac{\bar{b}_{0} \pm \sqrt{\bar{b}_{0}^{2}-4 \bar{a}_{0} \bar{c}_{0}}}{2 \bar{a}_{0}}\right)
$$

For the case $\theta_{i}<\theta_{c}$, the term $\cos \theta_{1}$ is real and $Z_{2}$ and $\beta_{2}$ are expressed as

$$
\begin{aligned}
& \beta_{2}=k_{\mathrm{o}} n_{2} \cos \theta_{1}=k_{\mathrm{o}} \sqrt{n_{2}^{2}-\bar{\beta}^{2}}, \quad \beta_{2} d_{2}=k_{0} \bar{w} d_{2}=\bar{W} \\
& Z_{2}=\left\{\begin{array}{l}
\frac{Z_{0} \bar{w}}{n_{2}^{2}}, \text { for } \| \text { polarization } \\
\frac{Z_{0}}{\bar{w}}, \text { for } \perp \text { polarization }
\end{array}\right.
\end{aligned}
$$

where

$$
\bar{w}=\sqrt{n_{2}^{2}-\bar{\beta}^{2}}
$$

The remaining terms $\bar{\beta}, u$, and $\mathrm{Z}_{1}$ remain unchanged and follow the same definitions as those for the case $\theta_{i}>\theta_{c}$ given by equations (2.16a), (2.16b), and (2.17) respectively. For this case $\mathrm{a}_{0}, \mathrm{~b}_{0}$, and $\mathrm{c}_{0}$ are given as 


$$
\begin{aligned}
& a_{0}=Z_{0} \hat{a}_{0} \\
& b_{0}=j Z_{0}{ }^{2} \hat{b}_{0} \\
& c_{0}=-Z_{0}{ }^{3} \hat{c}_{0}
\end{aligned}
$$

where

$$
\begin{aligned}
& \hat{a}_{\mathrm{o}}=\left\{\begin{array}{l}
\left(\frac{u}{n_{1}^{2}}\right) \tan (\bar{W})+\left(\frac{\bar{w}}{n_{2}^{2}}\right) \tan (U), \text { for } \| \text { polarization } \\
\left(\frac{1}{u}\right) \tan (\bar{W})+\left(\frac{1}{\bar{w}}\right) \tan (U), \text { for } \perp \text { polarization }
\end{array}\right. \\
& \hat{b}_{\mathrm{o}}=\left\{\begin{array}{l}
{\left[\left(\frac{u}{n_{1}{ }^{2}}\right)^{2}-\left(\frac{\bar{w}}{n_{2}{ }^{2}}\right)^{2}\right] \tan (U) \tan (\bar{W}), \text { for } \| \text { polarization }} \\
{\left[\left(\frac{1}{u}\right)^{2}-\left(\frac{1}{\bar{w}}\right)^{2}\right] \tan (U) \tan (\bar{W}), \text { for } \perp \text { polarization }}
\end{array}\right. \\
& \hat{c}_{\mathrm{o}}=\left\{\begin{array}{l}
\left(\frac{u \bar{w}}{n_{1} n_{2}^{2}}\right)\left[\left(\frac{u}{n_{1}^{2}}\right) \tan (U)+\left(\frac{\bar{w}}{n_{2}{ }^{2}}\right) \tan (\bar{W})\right], \text { for } \| \text { polarization } \\
\left(\frac{1}{u w}\right)\left[\left(\frac{1}{u}\right) \tan (U)+\left(\frac{1}{\bar{w}}\right) \tan (\bar{W})\right], \text { for } \perp \text { polarization }
\end{array}\right.
\end{aligned}
$$

Finally,

$$
Z_{\text {in }}=Z_{0}\left(\frac{-j \hat{b}_{0} \pm \sqrt{-\left(\hat{b}_{0}\right)^{2}+4 \hat{a}_{0} \hat{c}_{0}}}{2 \hat{a}_{0}}\right)
$$




\section{Chapter 3. Analysis of Planar 1-D Photonic Crystal Waveguide with Infinite Cladding}

Using the results for the reflection coefficient of a plane-wave incident on a semiinfinite periodic dielectric structure, propagation properties of guided modes in a planar one-dimensional photonic crystal waveguide are studied. First, the characteristic equations of guided modes are derived using the 'transverse resonance condition'. Then, complete solutions for the electric and magnetic field components of the modes are presented. The analysis of similar waveguides with a finite number of cladding layers is also carries out. Such waveguides, when the number of cladding layers is large, accurately approximate the waveguides with infinite layers in the cladding. The latter analysis allows us to validate the results for the infinite cladding photonic crystal waveguide obtained from the impedance approach.

\subsection{Characteristic Equations}

When the input impedance, $Z_{i n}$, seen at the interface of a semi-infinite periodic dielectric structure is calculated, the reflection coefficient can be found from (2.7). Having a dielectric layer of thickness $2 a$ sandwiched between two such semi-infinite periodic dielectric regions, a one-dimensional photonic crystal waveguide with an index profile as that shown in Figure 2.1 is formed. Figure 3.1 shows the front view of this waveguide. The characteristic equations for guided modes are obtained using the transverse resonance condition. This condition states that the total phase change in the transverse direction in the core region (i.e., along the x-axis in Figure 3.1) for one complete cycle of a ray representing the mode must be an interval multiple of $2 \pi$. This condition, in fact, is the requirement for constructive interference of the ray with itself after reflection. Mathematically, this condition is expressed as [23], 


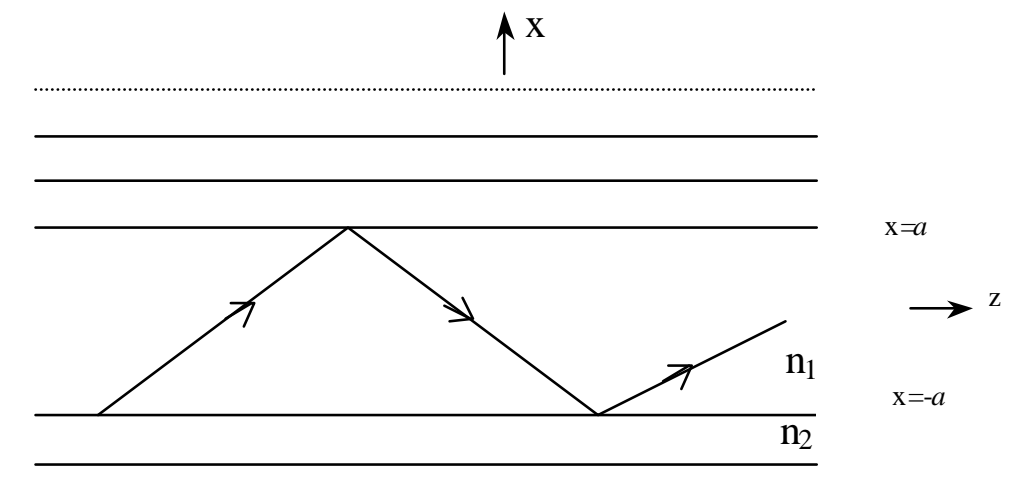

Figure 3.1 Front view of 1D planar photonic crystal waveguide

$$
-2 k_{0} \int_{-a}^{a} \sqrt{n_{1}{ }^{2}-\overline{\boldsymbol{\beta}}^{2}} d x+2 \delta_{\Gamma}=2 m \pi,
$$

where $m$ is an integer, $\delta_{\Gamma}$ is the phase of the reflection coefficient $\Gamma$ at $\mathrm{x}=a$ or $\mathrm{x}=-a$, $k_{0}$ is defined in (2.6), and $\bar{\beta}$ is the effective mode index (also referred to as the normalized propagation constant) defined in (2.16a). The mode index $\bar{\beta}$ is related to the mode propagation constant $\beta$ through $\bar{\beta}=\beta / k_{0}$. The integral term in (3.1) is easily evaluated, yielding

$$
-2 k_{0} \int_{-a}^{a} \sqrt{n_{1}^{2}-\overline{\boldsymbol{\beta}}^{2}} d x=-4 k_{0} u_{0} a=-4 U_{0},
$$

where $U_{0}=k_{0} u_{0} a$. To obtain $\delta_{\Gamma}$, first the expression for $\Gamma$ is determined from $\Gamma=\left(Z_{i n}-Z_{1}\right) /\left(Z_{i n}+Z_{1}\right)$, in which $Z_{i n}$ is substituted from (2.27) for $\boldsymbol{\theta}_{i}>\boldsymbol{\theta}_{c}$ and from (2.37) for $\boldsymbol{\theta}_{i}<\boldsymbol{\theta}_{c}$ and $Z_{1}$ is given in (2.17). Accordingly, for the case $\boldsymbol{\theta}_{i}>\boldsymbol{\theta}_{c}$, corresponding to $n_{2}<\bar{\beta}<n_{1} ; n_{1}>n_{2}$, the reflection coefficient is written as 


$$
\Gamma=\frac{-j\left(\frac{\bar{b}_{0} \pm \sqrt{\bar{b}_{0}^{2}-4 \bar{a}_{0} \bar{c}_{0}}}{2 \bar{a}_{0}}\right)-\bar{Z}_{1}}{-j\left(\frac{\bar{b}_{0} \pm \sqrt{\bar{b}_{0}^{2}-4 \bar{a}_{0} \bar{c}_{0}}}{2 \bar{a}_{0}}\right)+\bar{Z}_{1}},
$$

where $\bar{Z}_{1}=Z_{1} / Z_{0}$. It should be noted that for this case the magnitude of $\Gamma$ is unity, implying total internal reflection at $\mathrm{x}=a$ and $\mathrm{x}=-a$, and thus can be expressed as $\Gamma=e^{-j \delta_{\Gamma}}=e^{-j 2 \delta}$, where

$$
\delta=\frac{\delta_{\Gamma}}{2}=\tan ^{-1}\left(\frac{\bar{Z}_{1}}{\frac{\bar{b}_{0} \pm \sqrt{\bar{b}_{0}^{2}-4 \bar{a}_{0} \bar{c}_{0}}}{2 \bar{a}_{0}}}\right) .
$$

Combining (3.1) to (3.4), the characteristic equation is now obtained as

$$
U_{0}+\delta+\frac{m \pi}{2}=0,
$$

or

$$
\tan \delta=-\tan \left(U_{\mathrm{o}}+\frac{m \pi}{2}\right) .
$$

Substituting for $\delta$ from (3.4) in (3.6), yields

$$
\left(\frac{\overline{\mathrm{Z}}_{1}}{\frac{\overline{\mathrm{b}}_{0} \pm \sqrt{\overline{\mathrm{b}}_{0}{ }^{2}-4 \overline{\mathrm{a}}_{0} \overline{\mathrm{c}}_{0}}}{2 \overline{\mathrm{a}}_{0}}}\right)=-\tan \left(\mathrm{U}_{\mathrm{o}}+\frac{\mathrm{m} \pi}{2}\right)
$$

or

$$
\frac{2 \bar{a}_{0} \bar{Z}_{1}}{-\tan \left(U_{\mathrm{o}}+\frac{m \pi}{2}\right)}=\bar{b}_{0} \pm \sqrt{\bar{b}_{0}^{2}-4 \bar{a}_{0} \bar{c}_{0}}
$$




$$
-\left[\frac{2 \bar{a}_{0} \bar{Z}_{1}}{\tan \left(U_{\mathrm{o}}+\frac{m \pi}{2}\right)}+\bar{b}_{0}\right]= \pm \sqrt{\bar{b}_{0}^{2}-4 \bar{a}_{0} \bar{c}_{0}} .
$$

Squaring both sides of (3.7) and after some manipulations, the characteristic equation can be cast into the following form,

$$
\left(\bar{a}_{0} \bar{Z}_{1}^{2}+\bar{c}_{0}\right)+\left(\bar{a}_{0} \bar{Z}_{1}^{2}-\bar{c}_{0}\right) \cos \left(2 U_{\mathrm{o}}+m \pi\right)+\bar{b}_{0} \bar{Z}_{1} \sin \left(2 U_{\mathrm{o}}+m \pi\right)=0 .
$$

Knowing the waveguide parameters $a, \mathrm{~d}_{1}, \mathrm{~d}_{2}, n_{1}$, and $n_{2}$, and given the wavelength of operation $\lambda$, the characteristic equation (3.8) becomes a function of only $\bar{\beta}$ which can be solved numerically. In general, there may be more than one solution at a given wavelength and for a given set of waveguide parameters. Each solution represents the eigenvalue of a guided mode. Once the normalized propagation constant of a mode is determined, complete field solutions are readily obtained as detailed in section 3.2.

Equation (3.8) is the characteristic equation for TE modes when $\bar{a}_{0}, \bar{b}_{0}$, and $\bar{c}_{0}$ corresponding to $\perp$ polarization in (2.22), (2.25), and (2.26) are used. It represents the characteristic equation of TM modes when $\bar{a}_{0}, \bar{b}_{0}$, and $\bar{c}_{0}$ corresponding to $\|$ polarization, the upper lines in (2.22), (2.25), and (2.26), are used. Furthermore, (3.8) with $\mathrm{m}=0$ (or any even number) gives the characteristic equations for even TE and TM modes, while for $\mathrm{m}=1$ (or any odd number) it gives the characteristic equations for odd TE and TM modes.

The derivation of the characteristic equations presented above has been for the case $\boldsymbol{\theta}_{i}>\boldsymbol{\theta}_{c}$. For the case of $\boldsymbol{\theta}_{i}<\boldsymbol{\theta}_{c}$ corresponding to $\bar{\beta}<n_{2}<n_{1}$, taking advantage of the similarity between equation (2.37) and equation (2.27), the characteristic equation is 
identical to (3.7) in format and is obtained by replacing $\bar{a}_{0}, \bar{b}_{0}$, and $\bar{c}_{0}$ with $\hat{a}_{0}, \hat{b}_{0}$, and $\hat{c}_{0}$. Accordingly, the characteristic equation for the case $\boldsymbol{\theta}_{i}<\boldsymbol{\theta}_{c}$ is expressed as

$$
-\left[\frac{2 \hat{a}_{0} Z_{1}}{\tan \left(U_{o}+\frac{m \pi}{2}\right)}+\hat{b}_{0}\right]= \pm \sqrt{\hat{b}_{0}^{2}-4 \hat{a}_{0} \hat{c}_{0}}
$$

where $\hat{a}_{0}, \hat{b}_{0}$, and $\hat{c}_{0}$ are given by (2.34), (2.35), and (2.36), respectively.

\subsection{Slab Waveguide as Special Case}

The symmetric slab waveguide may be viewed as a special case of the planar waveguide with infinite periodic cladding in the limit of $d_{1} \rightarrow 0$. By setting $d_{1}=0$, the periodic cladding simply reduces to a region of constant refractive index $n_{2}$. Retrieving the characteristic equations of slab waveguide form (3.7) assures us of the correctness of the analysis presented in section 3.1. When $d_{1}=0$, it can be noted from equation (2.19) that $\mathrm{U}=0$ and then $\overline{\mathrm{b}}_{0}=0$. Moreover, from equations (2.22) and (2.26), when $\tan \mathrm{U}=0$, the coefficients $\bar{a}_{0}$ and $\bar{c}_{0}$ reduce to the following,

$$
\begin{gathered}
\bar{a}_{0}=\left\{\begin{array}{l}
\left(\frac{u}{n_{1}^{2}}\right) \tanh (W), \text { for } \| \text { polarization } \\
\left(\frac{1}{u}\right) \tanh (W), \text { for } \perp \text { polarization }
\end{array}\right. \\
{\overline{c_{0}}}=\left\{\begin{array}{l}
-\left(\frac{u w}{n_{1}^{2} n_{2}^{4}}\right)\left(\frac{w}{n_{2}^{2}}\right) \tanh (W), \text { for } \| \text { polarization } \\
-\left(\frac{1}{u w^{2}}\right) \tanh h(W), \text { for } \perp \text { polarization }
\end{array}\right.
\end{gathered}
$$

Substituting for $\bar{a}_{0}, \bar{b}_{0}$, and $\bar{c}_{0}$ in (3.7) with '-` sign, yields 


$$
\begin{aligned}
& {\left[\frac{2\left(\frac{u}{n_{1}{ }^{2}}\right)^{2}}{\tan \left(U_{0}+\frac{m \pi}{2}\right)}-2\left(\frac{u w}{n_{1}{ }^{2} n_{2}{ }^{2}}\right)\right] \tanh (w)=0, \text { forTM modes }(\| \text { Polarization })} \\
& {\left[\frac{2\left(\frac{1}{u}\right)^{2}}{\tan \left(U_{0}+\frac{m \pi}{2}\right)}-2\left(\frac{1}{u w}\right)\right] \tanh (w)=0, \text { forTE modes }(\perp \text { Polarization }) \text {. }}
\end{aligned}
$$

Since $\tanh (\mathrm{W}) \neq 0$, equations (3.12) and (3.13) reduce to

$$
\begin{aligned}
& U_{0} \cot \left(U_{\mathrm{o}}+\frac{m \pi}{2}\right)=\frac{n_{1}^{2}}{n_{2}^{2}} W_{0}, \text { for TM modes, } \\
& U_{0} \tan \left(U_{\mathrm{o}}+\frac{m \pi}{2}\right)=W_{0}, \text { for TE modes, }
\end{aligned}
$$

where $W_{0}=w d_{0}$. Equations (3.14) and (3.15) represent even TM and TE mode when $\mathrm{m}=0$ and odd TM and TE mode when $\mathrm{m}=1$. The above analysis confirms that equation (3.7) does reduce to the characteristic equations of a symmetric slab waveguide in the limit of $\mathrm{d}_{1} \rightarrow 0$.

\subsection{Field Analysis}

The electromagnetic fields of guided modes are solutions of Maxwell's equations subject to boundary conditions. Because the planar waveguide under investigation, as defined by the index profile shown in Figure 2.1, does not vary along the y-axis, the field solutions are independent of the y coordinate. Furthermore, assuming that propagation of guided modes occurs along the positive z-direction, the z-dependence of the fields is as $\exp (-j \beta \mathrm{z})$, where $\beta$ is the axial propagation constant. Therefore, it remains only the $\mathrm{x}$ 
dependence of the fields to be determined. The situation is similar to the simple slab waveguide where field solutions are obtained from a one-dimensional wave equation [24]. As in a slab waveguide, the solutions can be classified as TE (transverse electric) and TM (transverse magnetic) modes. For TE modes, the nonzero field components are $E_{\mathrm{y}}, H_{\mathrm{z}}$, and $H_{\mathrm{x}}$, while for $\mathrm{TM}$ mode the nonzero components include $H_{\mathrm{y}}, E_{\mathrm{z}}$, and $E_{\mathrm{x}}$. Complete field solutions can be obtained if $H_{\mathrm{z}}$ for TE modes and $E_{\mathrm{z}}$ for TM modes are determined, because in uniform waveguides transverse field components can be expressed in terms of axial field components [24].

\subsubsection{TE Modes}

We proceed with the calculation of fields for TE modes first. For these modes, the axial component of magnetic field is expressed as $H_{\mathrm{z}}=h_{\mathrm{z}}(\mathrm{x}) \cdot \exp (-\mathrm{j} \beta \mathrm{z})$, then $h_{\mathrm{z}}$ is governed by the following one-dimensional Helmholtz equation [24].

$$
\frac{d^{2} h_{z}}{d x^{2}}+T h_{z}=0
$$

Here, $T=k_{0}^{2} n^{2}-\beta^{2}$ and $n$ is set equal to $n_{1}$ or $n_{2}$ according to index profile of Figure 2.1. Once $h_{\mathrm{z}}$ is determined, solutions for other field components can be readily obtained from the following relations [24],

$$
\begin{aligned}
& E_{y}=\left(\frac{j \omega \mu_{0}}{T}\right) \frac{\partial H_{z}}{\partial x}, \\
& H_{x}=\left(-\frac{j \beta}{T}\right) \frac{\partial H_{z}}{\partial x} .
\end{aligned}
$$

Equation (3.16) is a simple second-order linear ordinary differential equation with constant coefficients. The solution of this equation is, in general, expressed as $h_{\mathrm{z}}=\mathrm{A} \cos (u \mathrm{x})+\mathrm{B} \sin (u \mathrm{x})$ in regions where $n=n_{1}$, and as $h_{\mathrm{z}}=\mathrm{A} \exp (|u| \mathrm{x})+\mathrm{B} \exp (-|u| \mathrm{x})$ in regions where $n=n_{2}$. In these solutions $u^{2}=T$. Since the geometry of the waveguide is symmetric, $h_{\mathrm{z}}$ (and similarly $e_{\mathrm{z}}$ for TM modes) in the central core region, $-a<\mathrm{x}<a$, can 
include only the sine or the cosine function. In other words, the solutions for TE modes (and likewise for TM modes) are separated into two groups: (i) the group for which the axial field component in the central core region includes only the sine function and thus the corresponding transverse field components include cosine functions are referred to as even TE (or TM) modes, (ii) the group for which the above field component includes only the cosine function and thus the corresponding transverse field components include sine functions are referred to as odd TE (or TM) modes. Simply put, transverse fields of even modes are even functions of $\mathrm{x}$, while those of the odd modes are odd functions of $\mathrm{x}$. The symmetry properties of the modes imply that field expressions in one half of the waveguide (say, the upper half) are sufficient. Based on the above considerations, the solution for the axial magnetic field component of TE modes in an $i$ th layer of the upper half region $(x>0)$ of the waveguide is expressed as,

$$
h_{z i}(x)=A_{i} Z_{i}\left(u_{i} x\right)+B_{i} \bar{Z}_{i}\left(u_{i} x\right), \quad x_{i-1}<x<x_{i}
$$

where $Z_{i}$ and $\bar{Z}_{i}$ are generalized functions defined as

$$
\begin{aligned}
& Z_{i}= \begin{cases}\sin , & i=\text { odd } \\
\frac{1}{\exp }, & i=\text { even }\end{cases} \\
& \bar{Z}_{i}= \begin{cases}\cos , & i=\text { odd } \\
\exp , & i=\text { even }\end{cases}
\end{aligned}
$$

and

$$
u_{i}=\sqrt{q_{i}\left(k_{0}{ }^{2} n_{i}^{2}-\beta^{2}\right)}
$$

with

$$
q_{i}=\left\{\begin{array}{l}
1, \quad i=\text { odd } \\
-1, \quad i=\text { even }
\end{array}\right.
$$




$$
n_{i}=\left\{\begin{array}{ll}
n_{1}, & i=\text { odd } \\
n_{2}, & i=\text { even }
\end{array},\right.
$$

In (3.19), $x_{i}$ in term of $a, d_{1}$, and $d_{2}$ is given as

$$
x_{i}=\left\{\begin{array}{l}
a+\frac{i}{2}\left(d_{1}+d_{2}\right)-d_{1}, \text { for } i=\text { even } \\
a+\frac{i-1}{2}\left(d_{1}+d_{2}\right), \text { for } i=\text { odd }
\end{array} ; i=1,2,3, \ldots\right.
$$

It is emphasized that for even TE modes $B_{1}=0$, while for odd TE modes $A_{1}=0$. Also, we choose to set $A_{1}=1$ for even TE modes and $B_{1}=1$ for odd TE modes.

The transverse field components are calculated from (3.17) and (3.18). Dropping the propagation term $\exp (-\mathrm{j} \beta \mathrm{z})$ from the solutions, these fields in the ith layer of the waveguide are calculated as

$$
\begin{aligned}
& e_{y i}=\frac{j \omega \mu_{0}}{k_{0}^{2} n_{i}^{2}-\beta^{2}} \frac{d h_{z i}}{d x}, \\
& h_{x i}=-\frac{\beta}{\omega \mu_{0}} e_{y i}
\end{aligned}
$$

Substituting for $h_{z i}$ from (3.19), we obtain

$$
e_{y i}=\frac{j \omega \mu_{0} q_{i}}{u_{i}}\left[A_{i} Z_{i}^{\prime}\left(u_{i} x\right)+B_{i} \bar{Z}_{i}^{\prime}\left(u_{i} x\right)\right], \quad x_{i-1}<x<x_{i} \text {, }
$$

where 


$$
\begin{gathered}
Z_{i}^{\prime}=\left\{\begin{array}{l}
\cos , \quad i=\text { odd } \\
-\frac{1}{\exp }, i=\text { even }
\end{array}=\left\{\begin{array}{ll}
\bar{Z}_{i}, & i=\text { odd } \\
-Z_{i}, & i=\text { even }
\end{array},\right.\right. \\
\bar{Z}_{i}^{\prime}=\left\{\begin{array}{l}
-\sin , \quad i=\text { odd } \\
\exp , i=\text { even }
\end{array}=\left\{\begin{array}{l}
-Z_{i}, \quad i=\text { odd } \\
\bar{Z}_{i}, i=\text { even }
\end{array}\right.\right.
\end{gathered}
$$

\subsubsection{Boundary Conditions}

To find the amplitude coefficients $A_{i}$ and $B_{i}$, boundary conditions are applied. The boundaries in the upper half region are $x=x_{i} ; i=1,2,3, \ldots$, where $x_{i}$ is defined in (3.25). Boundary conditions for dielectric interfaces amount to continuity of tangential components of electric and magnetic fields. For TE modes, tangential components include $e_{y}$ and $h_{z}$. Thus, boundary conditions at an interface between $i$ th and $(i+1)$ th layers are written as

$$
\begin{aligned}
& h_{z i}\left(x=x_{i}\right)=h_{z},_{i+1}\left(x=x_{i}\right), \\
& e_{y i}\left(x=x_{i}\right)=e_{y,{ }_{i+1}}\left(x=x_{i}\right) .
\end{aligned}
$$

Imposing the above conditions, we have

$$
\begin{aligned}
& A_{i} Z_{i}\left(u_{i} x_{i}\right)+B_{i} \bar{Z}_{i}\left(u_{i} x_{i}\right)=A_{i+1} Z_{i+}\left(u_{i+1} x_{i}\right)+B_{i+1} \bar{Z}_{i+1}\left(u_{i+1} x_{i}\right) \\
& \frac{j \omega \mu_{0} q_{i}}{u_{i}}\left[A_{i} Z_{i}^{\prime}\left(u_{i} x_{i}\right)+B_{i} \bar{Z}_{i}^{\prime}\left(u_{i} x_{i}\right)\right]=\frac{j \omega \mu_{0} q_{i+1}}{u_{i+1}}\left[A_{i+1} Z_{i+1}^{\prime}\left(u_{i+1} x_{i}\right)+B_{i+1} \bar{Z}_{i+1}^{\prime}\left(u_{i+1} x_{i}\right)\right]
\end{aligned}
$$

Introducing 


$$
\begin{aligned}
& U_{i}=u_{i} x_{i}, \\
& W_{i}=u_{i+1} x_{i},
\end{aligned}
$$

the boundary conditions in (3.32) are written as

$$
\begin{aligned}
& A_{i} Z_{i}\left(U_{i}\right)+B_{i} \bar{Z}_{i}\left(U_{i}\right)=A_{i+1} Z_{i+1}\left(W_{i}\right)+B_{i+1} \bar{Z}_{i+1}\left(W_{i}\right), \\
& \left(\frac{u_{i+1}}{u_{i}}\right)\left(\frac{q_{i}}{q_{i+1}}\right)\left[A_{i} Z_{i}^{\prime}\left(U_{i}\right)+B_{i} \bar{Z}_{i}^{\prime}\left(U_{i}\right)\right]=A_{i+1} Z_{i+1}^{\prime}\left(W_{i}\right)+B_{i+1} \bar{Z}_{i+1}^{\prime}\left(W_{i}\right) .
\end{aligned}
$$

Equations (3.35a) and (3.35b) may be regarded as a system of two equations and two unknowns in which $A_{i+1}$ and $B_{i+1}$ are unknown, while $A_{i}$ and $B_{i}$ are treated as known quantities. Solving for $A_{i+1}$ and $B_{i+1}$, yields

$$
\begin{aligned}
& A_{i+1}=a_{11}{ }^{i} A_{i}+a_{12}{ }^{i} B_{i}, \\
& B_{i+1}=a_{21}{ }^{i} A_{i}+a_{22}{ }^{i} B_{i},
\end{aligned}
$$

or

$$
\left[\begin{array}{l}
A_{i+1} \\
B_{i+1}
\end{array}\right]=\left[\begin{array}{ll}
a_{11}{ }^{i} & a_{12}{ }^{i} \\
a_{21}{ }^{i} & a_{22}{ }^{i}
\end{array}\right]\left[\begin{array}{l}
A_{i} \\
B_{i}
\end{array}\right] .
$$

where

$$
a_{11}{ }^{i}= \begin{cases}\frac{1}{2}\left[\sin \left(U_{i}\right)+\frac{W_{i}}{U_{i}} \cos \left(U_{i}\right)\right] e^{W_{i}}, & i=\text { odd } \\ {\left[\sin \left(W_{i}\right)+\frac{W_{i}}{U_{i}} \cos \left(W_{i}\right)\right] e^{-U_{i}},} & i=\text { even }\end{cases}
$$




$$
\begin{aligned}
& a_{12}{ }^{i}= \begin{cases}\frac{1}{2}\left[\cos \left(U_{i}\right)-\frac{W_{i}}{U_{i}} \sin \left(U_{i}\right)\right] e^{W_{i}}, & i=\text { odd } \\
{\left[\sin \left(W_{i}\right)-\frac{W_{i}}{U_{i}} \cos \left(W_{i}\right)\right] e^{+U_{i}},} & i=\text { even }\end{cases} \\
& a_{21}{ }^{i}= \begin{cases}\frac{1}{2}\left[\sin \left(U_{i}\right)-\frac{W_{i}}{U_{i}} \cos \left(U_{i}\right)\right] e^{-W_{i}}, & i=\text { odd } \\
{\left[\cos \left(W_{i}\right)-\frac{W_{i}}{U_{i}} \sin \left(W_{i}\right)\right] e^{-U_{i}},} & i=\text { even }\end{cases} \\
& a_{22}{ }^{i}= \begin{cases}\frac{1}{2}\left[\cos \left(U_{i}\right)+\frac{W_{i}}{U_{i}} \sin \left(U_{i}\right)\right] e^{-W_{i}}, & i=\text { odd } \\
{\left[\cos \left(W_{i}\right)+\frac{W_{i}}{U_{i}} \sin \left(W_{i}\right)\right] e^{U_{i}},} & i=\text { even }\end{cases}
\end{aligned}
$$

\subsubsection{TM Modes}

TM modes can be treated in a manner similar to TE modes. The axial field component for these modes is $E_{\mathrm{z}}=e_{\mathrm{z}}(\mathrm{x}) \cdot \exp (-\mathrm{j} \beta \mathrm{z})$, where $e_{\mathrm{z}}$ is the solution of the onedimensional wave equation,

$$
\frac{d^{2} e_{z}}{d x^{2}}+T e_{z}=0
$$

Other field components can be obtained using the following relations [24],

$$
\begin{aligned}
& H_{y}=-\frac{j \omega \varepsilon_{0} n^{2}}{T} \frac{\partial E_{z}}{\partial x}, \\
& E_{x}=\frac{j \beta}{T} \frac{\partial E_{z}}{\partial x} .
\end{aligned}
$$


The solution for $e_{\mathrm{z}}$ in an $i$ th layer of the upper half region $(\mathrm{x}>0)$ of the waveguide is expressed as,

$$
e_{z i}(x)=A_{i} Z_{i}\left(u_{i} x\right)+B_{i} \bar{Z}_{i}\left(u_{i} x\right), \quad x_{i-1}<x<x_{i} .
$$

where $Z_{i}, \bar{Z}_{i}, u_{i}, x_{i}$ follow the same definitions as those given for TE modes.

The transverse field components are calculated from (3.41) and (3.42). Dropping the propagation term $\exp (-\mathrm{j} \beta \mathrm{z})$ from the solutions, these fields in the $i$ th layer of the waveguide are obtained from

$$
\begin{aligned}
& h_{y i}=\frac{-j \omega \varepsilon_{0} n_{i}^{2}}{k_{0}^{2} n_{i}^{2}-\beta^{2}} \frac{d e_{z i}}{d x}, \\
& e_{x i}=-\frac{\omega \varepsilon_{0} n_{i}^{2}}{\beta} h_{y i .}
\end{aligned}
$$

Substituting for $e_{z i}$ from (3.43), the tangential component of the magnetic field is obtained as

$$
h_{y i}=\frac{-j \omega \varepsilon_{0} n_{i}^{2}}{u_{i}}\left[A_{i} Z_{i}^{\prime}\left(u_{i} x\right)+B_{i} \bar{Z}_{i}^{\prime}\left(u_{i} x\right)\right], \quad x_{i-1}<x<x_{i} .
$$

As in the case of TE modes, here too for even TM modes $B_{1}=0, A_{1}=1$, while for odd TM modes $A_{1}=0, B_{1}=1$. The boundary conditions at the interface between $i$ th and $(i+1)$ th layers are obtained from the continuity of $e_{\mathrm{z}}$ and $h_{\mathrm{y}}$. That is,

$$
\begin{aligned}
& e_{z i}\left(x=x_{i}\right)=e_{z}{ }_{i+1}\left(x=x_{i}\right), \\
& h_{y i}\left(x=x_{i}\right)=h_{y, i+1}\left(x=x_{i}\right) .
\end{aligned}
$$


Now, following the same steps as in TE modes, $A_{i+1}$ and $B_{i+1}$ are expressed in terms of $A_{i}$ and $B_{i}$. Using the matrix form given by (3.38), the entries for TM modes are as follows,

$$
\begin{aligned}
& a_{11}{ }^{i}= \begin{cases}\frac{1}{2}\left[\sin \left(U_{i}\right)+\frac{n_{i}{ }^{2}}{n_{i+1}} \frac{W_{i}}{U_{i}} \cos \left(U_{i}\right)\right] e^{W_{i}}, & i=\text { odd } \\
{\left[\sin \left(W_{i}\right)+\frac{n_{i}{ }^{2}}{n_{i+1}{ }^{2}} \frac{W_{i}}{U_{i}} \cos \left(W_{i}\right)\right] e^{-U_{i}},} & i=\text { even }\end{cases} \\
& a_{12}{ }^{i}= \begin{cases}\frac{1}{2}\left[\cos \left(U_{i}\right)-\frac{n_{i}{ }^{2}}{n_{i+1}^{2}} \frac{W_{i}}{U_{i}} \sin \left(U_{i}\right)\right] e^{W_{i}}, & i=\text { odd } \\
{\left[\sin \left(W_{i}\right)-\frac{n_{i}{ }^{2}}{n_{i+1}^{2}} \frac{W_{i}}{U_{i}} \cos \left(W_{i}\right)\right] e^{+U_{i}},} & i=\text { even }\end{cases} \\
& a_{21}{ }^{i}= \begin{cases}\frac{1}{2}\left[\sin \left(U_{i}\right)-\frac{n_{i}{ }^{2}}{n_{i+1}^{2}} \frac{W_{i}}{U_{i}} \cos \left(U_{i}\right)\right] e^{-W_{i}}, & i=\text { odd } \\
{\left[\cos \left(W_{i}\right)-\frac{n_{i}{ }^{2}}{n_{i+1}^{2}} \frac{W_{i}}{U_{i}} \sin \left(W_{i}\right)\right] e^{-U_{i}},} & i=\text { even }\end{cases} \\
& a_{22}{ }^{i}= \begin{cases}\frac{1}{2}\left[\cos \left(U_{i}\right)+\frac{n_{i}{ }^{2}}{n_{i+1}^{2}} \frac{W_{i}}{U_{i}} \sin \left(U_{i}\right)\right] e^{-W_{i}}, & i=\text { odd } \\
{\left[\cos \left(W_{i}\right)+\frac{n_{i}{ }^{2}}{n_{i+1}^{2}} \frac{W_{i}}{U_{i}} \sin \left(W_{i}\right)\right] e^{U_{i}},} & i=\text { even }\end{cases}
\end{aligned}
$$

Finally, after finding the normalized propagation constant $\bar{\beta}$ for a particular mode, as was discussed in the analysis presented in section 3.1, and knowing waveguide parameters $n_{1}, n_{2}, a_{0}, x_{1}, x_{2}, \ldots$ and $\mathrm{A}_{1}=1, \mathrm{~B}_{1}=0$ for even TE and TM or $\mathrm{A}_{1}=0, \mathrm{~B}_{1}=1$ for odd TE and TM modes, $\left(\mathrm{A}_{2}, \mathrm{~B}_{2}\right)$, then $\left(\mathrm{A}_{3}, \mathrm{~B}_{3}\right)$, etc. can be calculated. Then, having determined field coefficients $A_{i}$ and $B_{i}$, complete field solutions become readily available. 


\subsubsection{Fields Solutions Corresponding to Photonic Crystal Effect}

For the case of $0<\bar{\beta}<\mathrm{n}_{2}$, field solutions can be obtained in a manner similar to case of $n_{1}>\bar{\beta}>n_{2}$. The only difference is that both terms in the field solutions are sinusoidal; that is, for TE modes, $h_{z i}(x)$ is still obtained from (3.19), but $Z_{i}$ and $\bar{Z}_{i}$ functions are now defined as

$$
\begin{aligned}
& Z_{i}=\sin , \quad i=1,2,3, \ldots . \\
& \bar{Z}_{i}=\cos , \quad i=1,2,3, \ldots \ldots
\end{aligned}
$$

and $u_{i}=\sqrt{k_{0}{ }^{2} n_{i}{ }^{2}-\beta^{2}}$. Imposing the same boundary conditions as before, the relationships between coefficients $A_{i+1}$ and $B_{i+1}$ and coefficients $A_{i}$ and $B_{i}$ are obtained as summarized below

$$
\begin{aligned}
& a_{11}{ }^{i}=\sin \left(U_{i}\right) \sin \left(W_{i}\right)+\frac{W_{i}}{U_{i}} \cos \left(U_{i}\right) \cos \left(W_{i}\right) \\
& a_{12}{ }^{i}=\cos \left(U_{i}\right) \sin \left(W_{i}\right)-\frac{W_{i}}{U_{i}} \sin \left(U_{i}\right) \cos \left(W_{i}\right) \\
& a_{21}{ }^{i}=\sin \left(U_{i}\right) \cos \left(W_{i}\right)-\frac{W_{i}}{U_{i}} \cos \left(U_{i}\right) \sin \left(W_{i}\right) \\
& a_{22}{ }^{i}=\cos \left(U_{i}\right) \cos \left(W_{i}\right)+\frac{W_{i}}{U_{i}} \sin \left(U_{i}\right) \sin \left(W_{i}\right)
\end{aligned}
$$

In a similar manner for TM modes, the following results are found,

$$
\begin{aligned}
& a_{11}{ }^{i}=\sin \left(U_{i}\right) \sin \left(W_{i}\right)+\frac{n_{i}{ }^{2}}{n_{i+1}^{2}} \frac{W_{i}}{U_{i}} \cos \left(U_{i}\right) \cos \left(W_{i}\right) \\
& a_{12}{ }^{i}=\cos \left(U_{i}\right) \sin \left(W_{i}\right)-\frac{n_{i}^{2}}{n_{i+1}^{2}} \frac{W_{i}}{U_{i}} \sin \left(U_{i}\right) \cos \left(W_{i}\right)
\end{aligned}
$$




$$
\begin{aligned}
& a_{21}{ }^{i}=\sin \left(U_{i}\right) \cos \left(W_{i}\right)-\frac{n_{i}^{2}}{n_{i+1}^{2}} \frac{W_{i}}{U_{i}} \cos \left(U_{i}\right) \sin \left(W_{i}\right) \\
& a_{22}{ }^{i}=\cos \left(U_{i}\right) \cos \left(W_{i}\right)+\frac{n_{i}{ }^{2}}{n_{i+1}^{2}} \frac{W_{i}}{U_{i}} \sin \left(U_{i}\right) \sin \left(W_{i}\right)
\end{aligned}
$$

\subsection{Waveguide with Finite Number of Cladding Layers}

To verify the characteristic equation and the propagation constant obtained from the analysis given for 1-D photonic crystal waveguide with infinite number of cladding layers presented in section 3.1, we obtain the characteristic equation for a waveguide with a finite number of $2 \mathrm{~N}+1$ layers (core $+\mathrm{N}$ cladding layers in the $\mathrm{x}>0$ region and $+\mathrm{N}$ cladding layers in the $\mathrm{x}<0$ region) [25]. Fields solutions in an $i$ th layer of the waveguide with finite number of cladding layers are identical to those in the waveguide with infinite cladding layers. Accordingly, all results in (3.16) to (3.48) remain valid, but this time the normalized propagation constant $\bar{\beta}$ is also unknown. An extra restriction on the solutions for the waveguide with finite number of cladding layers is that the fields in the regions $x>\left|x_{N}\right|$ must be exponentially decaying. To maintain this restriction, one concludes from (3.19) that $B_{N+1}=0$. This requirement can be exploited in determining a characteristic equation from which the propagation constant can be found. In doing so, (3.38) is used repeatedly such that the field coefficients for the Nth cladding layer $\left(A_{N+1}, B_{N+1}\right)$ are expressed in terms of the field coefficients for the central core region $\left(A_{1}, B_{1}\right)$. The results is

$$
\begin{aligned}
& {\left[\begin{array}{l}
A_{N+1} \\
B_{N+1}
\end{array}\right]=\left[\begin{array}{ll}
a_{11}{ }^{N} & a_{12}{ }^{N} \\
a_{21}{ }^{N} & a_{22}{ }^{N}
\end{array}\right]\left[\begin{array}{ll}
a_{11}{ }^{N} & a_{12}{ }^{N} \\
a_{21}{ }^{N} & a_{22}{ }^{N}
\end{array}\right] \cdots\left[\begin{array}{ll}
a_{11}{ }^{2} & a_{12}{ }^{2} \\
a_{21}{ }^{2} & a_{22}{ }^{2}
\end{array}\right]\left[\begin{array}{ll}
a_{11} & a_{12}{ }^{1} \\
a_{21} & a_{22}{ }^{1}
\end{array}\right]\left[\begin{array}{l}
A_{1} \\
B_{1}
\end{array}\right],} \\
& =\left[\begin{array}{ll}
b_{11} & b_{12} \\
b_{21} & b_{22}
\end{array}\right]\left[\begin{array}{l}
A_{1} \\
B_{1}
\end{array}\right],
\end{aligned}
$$


where

$$
\left[\begin{array}{ll}
b_{11} & b_{12} \\
b_{21} & b_{22}
\end{array}\right]=\prod_{i=1}^{N}\left[\begin{array}{ll}
a_{11}{ }^{i} & a_{12}{ }^{i} \\
a_{21}{ }^{i} & a_{22}{ }^{i}
\end{array}\right] \text {. }
$$

Therefore,

$$
\left[\begin{array}{l}
A_{N+1} \\
B_{N+1}
\end{array}\right]=\left[\begin{array}{ll}
b_{11} & b_{12} \\
b_{21} & b_{22}
\end{array}\right]\left[\begin{array}{l}
A_{1} \\
B_{1}
\end{array}\right] .
$$

Substituting $B_{\mathrm{N}+1}=0$, and $B_{1}=0$ and $A_{1}=1$ for even modes, the characteristic equation of these modes is obtained as

$$
\mathrm{b}_{21}=0 \text {. }
$$

Clearly, $b_{21}$ is dependent on $a_{11}^{i}, a_{12}^{i}, a_{21}^{i}$, and $a_{22}^{i} ; i=1,2,3, \ldots \mathrm{N}$, which are given by (3.39a) - (3.39d) for TE modes and by (3.48a) - (3.48d) for TM modes. Thus, (3.56) includes the characteristic equations for both even TE and TM modes. Similarly, using $B_{\mathrm{N}+1}=0$, and $B_{1}=1$ and $A_{1}=0$ for odd modes, the characteristic equation for odd modes is obtained as

$$
b_{22}=0 \text {. }
$$

Here too, (57) includes the characteristic equations for both odd TE and TM modes. Knowing that $a_{11}^{i}, a_{12}^{i}, a_{21}^{i}$, and $a_{22}^{i}$ are all functions of waveguide parameters $a, \mathrm{~d}_{1}, \mathrm{~d}_{2}, n_{1}$, and $n_{2}$, wavelength of operation $\lambda$, and normalized propagation constant $\bar{\beta}$, thus for given waveguide parameters and source wavelength (3.56) and (3.57) can be solved for $\bar{\beta}$. The numerical results for propagation characteristics and field plots are presented in the following chapter. 


\section{Chapter 4. Numerical Results}

In chapter 2, the reflection coefficient for a plane wave incident upon a semiinfinite periodic dielectric structure was analyzed. Various cases corresponding to perpendicular and parallel polarizations as well as the angle of incidence greater and smaller than the critical angle were considered. The reflection coefficient was expressed in terms of a wave impedance $Z_{\text {in }}$ given by (2.27) or (2.37). In chapter 3, exact analytical solutions of guided modes in a one-dimensional planar photonic crystal waveguide, consisting of a dielectric core region sandwiched between two semi-infinite periodic dielectric structures, were presented. In this chapter, numerical results for the wave impedance associated with reflection from a semi-infinite periodic dielectric structure, propagation characteristics of guided modes in one-dimensional photonic crystal waveguides, and the field plots for such modes are presented. The primary objective is to demonstrate that in addition to the well known total internal reflection effect, another phenomenon referred to as photonic crystal effect can contribute to guidance of electromagnetic waves.

\subsection{Wave Impedance}

The wave impedance $Z_{i n}$ is obtained from (2.27) when $\boldsymbol{\theta}_{i}>\boldsymbol{\theta}_{c}$ and from (3.37) when $\boldsymbol{\theta}_{i}<\boldsymbol{\theta}_{c}$, where $\boldsymbol{\theta}_{c}=\sin ^{-1}\left(n_{2} / n_{1}\right)$. Let us consider a periodic dielectric structure with a geometry as that shown in Figure (2.3). As an example case, the parameters of the structure are chosen as follows: $n_{1}=1.5, n_{2}=1, d_{1}=1.5 \mu \mathrm{m}$, and $d_{2}=1.0 \mu \mathrm{m}$. For this example the critical angle is $\theta_{c}=41.81^{\circ}$. The normalized wave impedance, $\bar{Z}_{i n}=Z_{i n} / Z_{0}$, is calculated at several wavelengths and for a range of incident angles $\boldsymbol{\theta}_{i}$. We consider four different wavelengths including $\lambda=0.5,1.0,1.5$, and $2.0 \mu \mathrm{m}$. The angle of incidence $\theta_{i}$ is varied between $5^{\circ}$ and $85^{\circ}$ in $1^{\circ}$ increment. The results for $\bar{Z}_{i n}$ are presented in two separate real and imaginary parts showing their variations versus the incident angle $\boldsymbol{\theta}_{i}$. 
For each real or imaginary part of $\bar{Z}_{\text {in }}$, four graphs, resulting from the choices of parallel or perpendicular polarization and a ' + ' or a '' sign in front of the square-root term in (2.27) and (2.28), are provided.

Figure 4.1 illustrates variations of the real part of $\bar{Z}_{i n}$ versus $\theta_{i}$ at four wavelengths for the case of parallel polarization and the '-' solution It is noted that when the angle of incidence $\theta_{i}$ exceeds the critical angle $\theta_{c}=41.81^{\circ}$ by few degrees or more the real part of $\bar{Z}_{i n}$ is zero; that is, the input impedance is purely imaginary. Figure 4.2 shows variations of the imaginary part of $\bar{Z}_{i n}$ for the structure as in Figure 4.1. It is seen that the imaginary part of $\bar{Z}_{i n}$ is primarily non-zero, thus for most incident angles $\boldsymbol{\theta}_{i}>\boldsymbol{\theta}_{c}$ where the real part of $\bar{Z}_{i n}$ is zero, the reflection coefficient $\Gamma$ given in (2.7) assumes a magnitude of unity; that is, $|\Gamma|=1$. This corresponds to the well known phenomenon of 'total internal reflection' which is the underlying principle for guidance of electromagnetic waves in conventional (not of photonic crystal type) dielectric waveguides and optical fibers. In conventional step-index dielectric waveguides, for $\theta_{i}<\theta_{c}$ the reflection coefficient at the core-cladding boundary has a magnitude less than unity, implying that electromagnetic waves radiate out of the core region and can no longer be guided. However, upon close examination of Figure 4.1, we can see that for incident angles below $\boldsymbol{\theta}_{c}$ there are few locations on the graphs where $\operatorname{Re}\left[\bar{Z}_{\text {in }}\right]=0$, leading to $|\Gamma|=1$ and therefore total reflection. This phenomenon is unique to photonic crystal structures and is due to complete constructive interference of multiply-reflected waves. For example, in Figure 4.1(b) there are two points between $\theta_{i}=20^{\circ}$ and $\theta_{i}=30^{\circ}$ at which $\operatorname{Re}\left[\bar{Z}_{i n}\right]=0$. Also, in Figure 4.1(d) for a range of incident angles $\theta_{i}<12^{\circ}$ the wave impedance is purely imaginary. The latter case, in fact, implies that even waves normally incident on a photonic crystal structure can be reflected $100 \%$ at certain wavelengths.

Results for the real and imaginary part of $\bar{Z}_{i n}$ for the case of perpendicular polarization and the '-' solution are presented in Figures 4.3 and 4.4, respectively. In this 


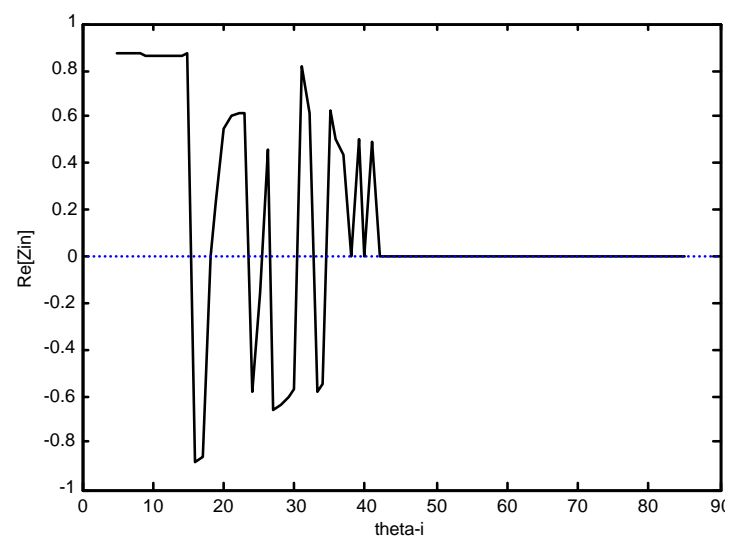

(a)

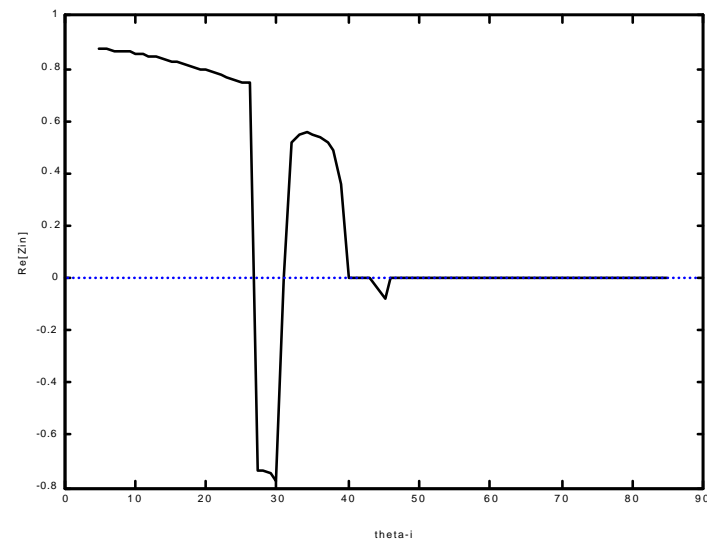

(c)

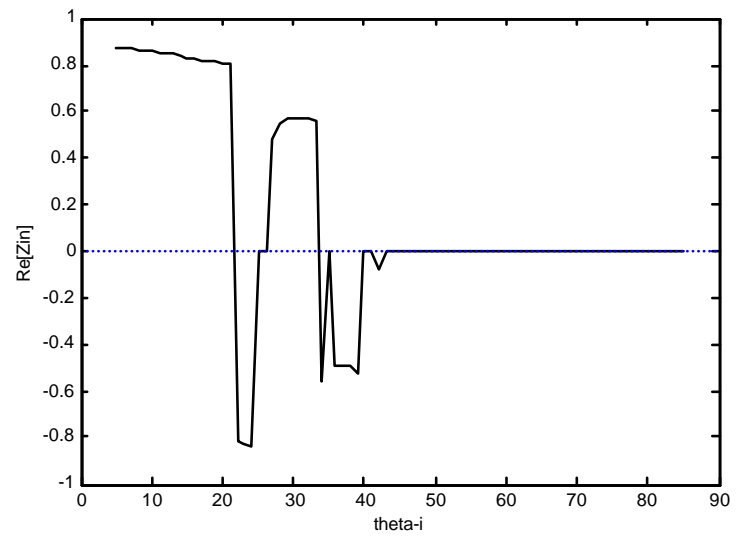

(b)

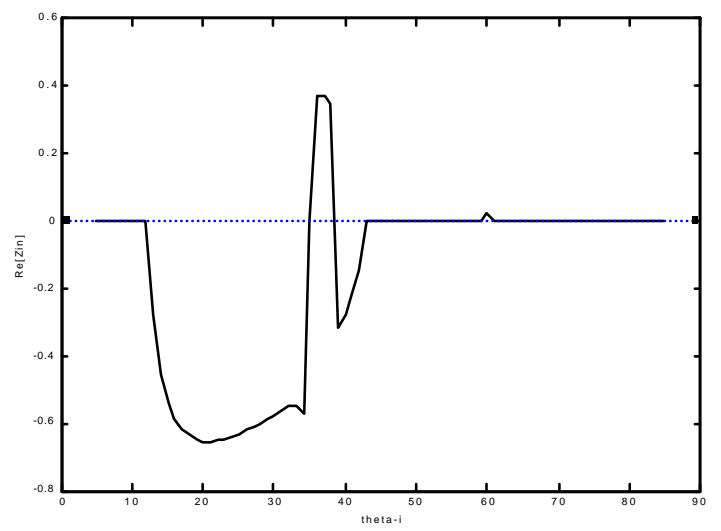

(d)

Figure 4.1 Variations of the real part of normalized wave impedance, $\operatorname{Re}\left[\bar{Z}_{i n}\right]$, versus incident angle $\theta_{i}$ for the case of '-' solution and parallel polarization at the wavelengths (a) $\lambda=0.5 \mu \mathrm{m}$, (b) $\lambda=1.0 \mu \mathrm{m}$, (c) $\lambda=1.5 \mu \mathrm{m}$, (d) $\lambda=2.0 \mu \mathrm{m}$. The parameters of the semi-infinite structure are $n_{1}=1.5, n_{2}=1, d_{1}=1.5 \mu \mathrm{m}$, and $d_{2}=1.0 \mu m$. 


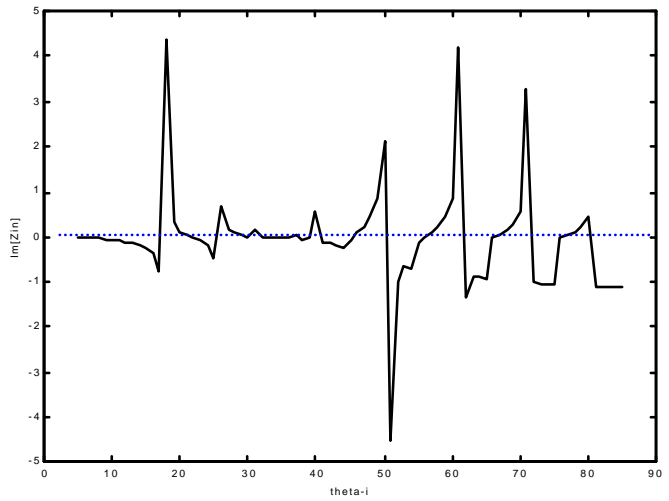

(a)

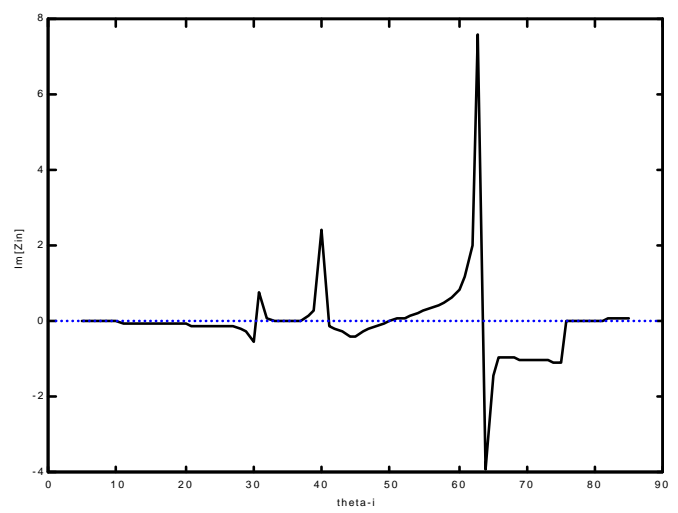

(c)

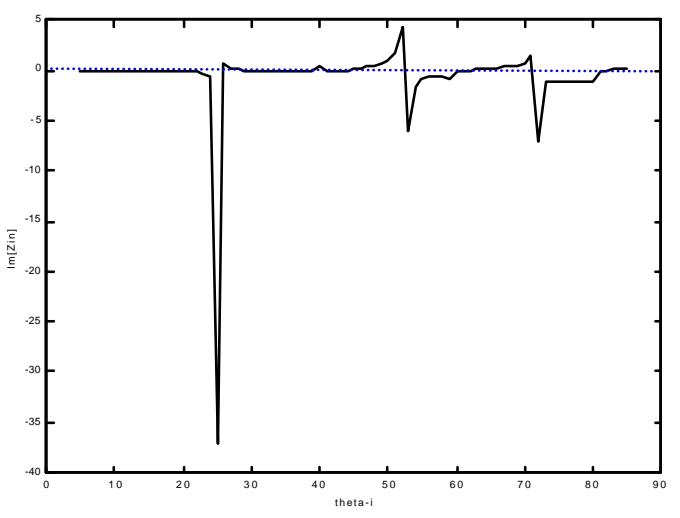

(b)

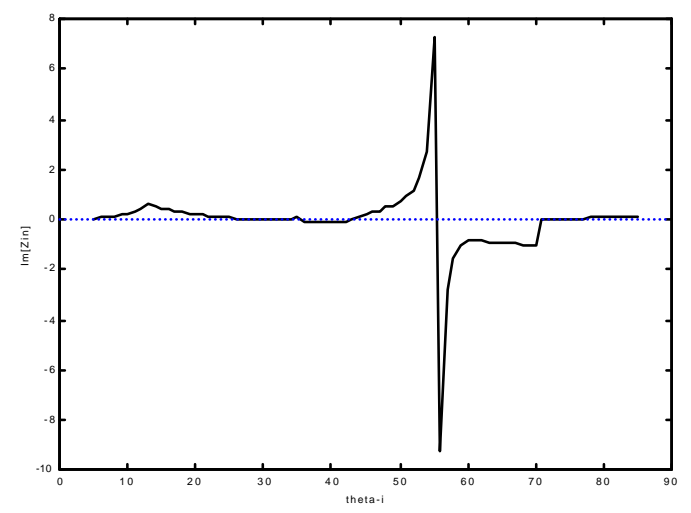

(d)

Figure 4.2 Variations of the imaginary part of normalized wave impedance, $\operatorname{Im}\left[\bar{Z}_{i n}\right]$, versus incident angle $\theta_{i}$ for the case of '-' solution and parallel polarization at the wavelengths (a) $\lambda=0.5 \mu \mathrm{m}$, (b) $\lambda=1.0 \mu \mathrm{m}$, (c) $\lambda=1.5 \mu \mathrm{m}$, (d) $\lambda=2.0$ $\mu \mathrm{m}$. The parameters of the structure are the same as those in Figure 4.1. 


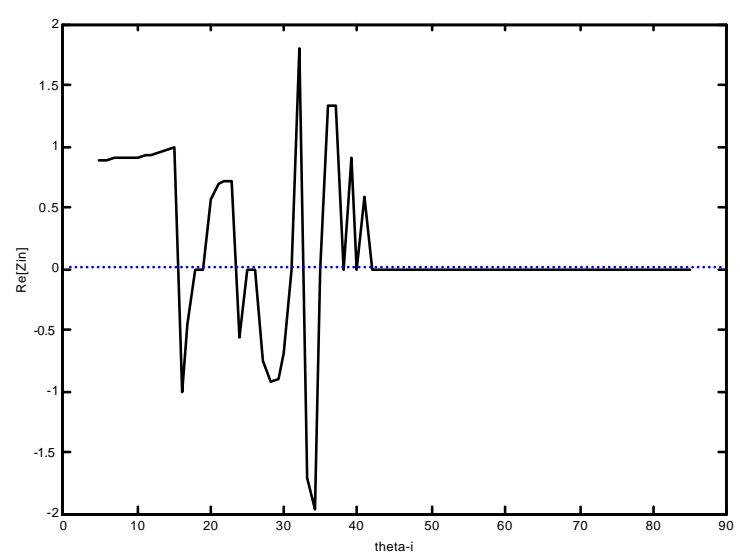

(a)

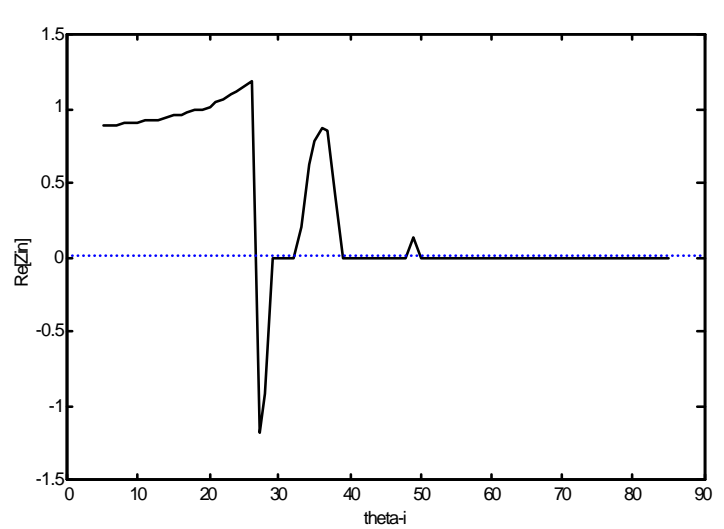

(c)

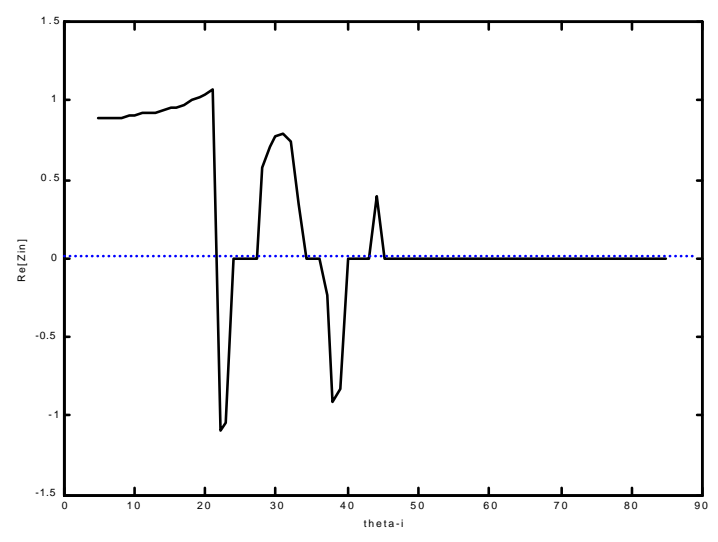

(b)

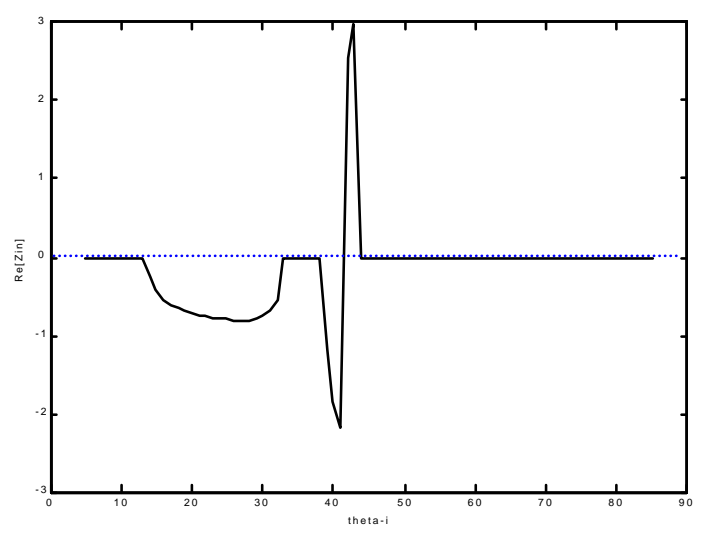

(d)

Figure 4.3 Variations of the real part of normalized wave impedance, $\operatorname{Re}\left[\bar{Z}_{i n}\right]$, versus incident angle $\theta_{i}$ for the case of '-' solution and perpendicular polarization at the wavelengths (a) $\lambda=0.5 \mu \mathrm{m}$, (b) $\lambda=1.0 \mu \mathrm{m}$, (c) $\lambda=1.5 \mu \mathrm{m}$, (d) $\lambda=2.0$ $\mu \mathrm{m}$. The parameters of the structure are the same as those in Figure 4.1. 


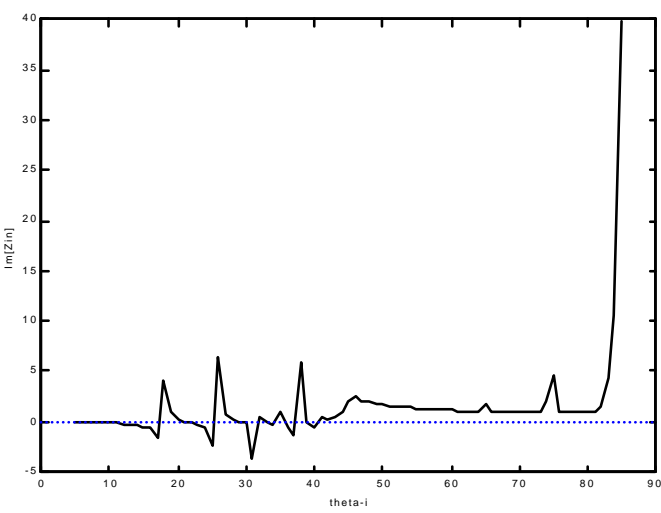

(a)

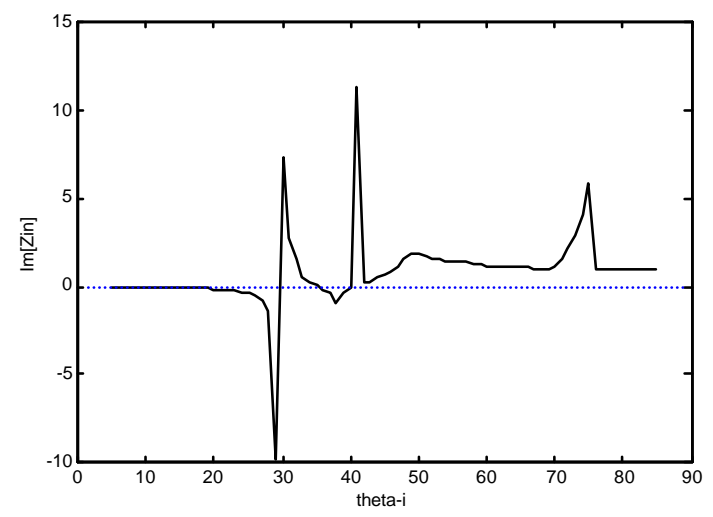

(c)

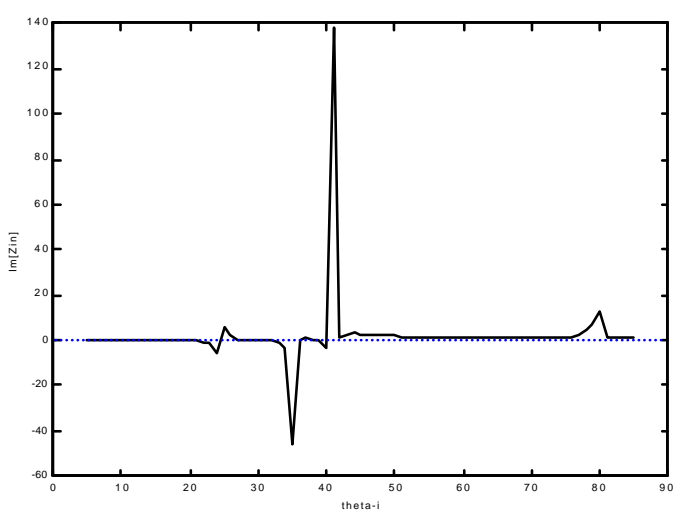

(b)

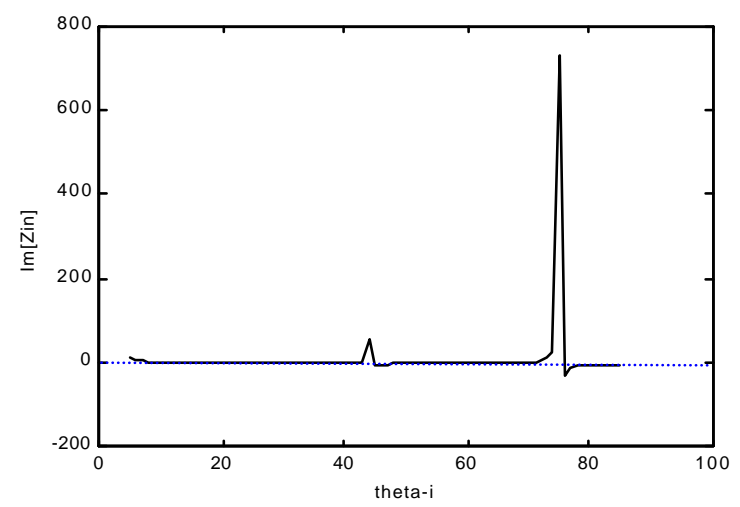

(d)

Figure 4.4 Variations of the imaginary part of normalized wave impedance, $\operatorname{Im}\left[\bar{Z}_{i n}\right]$, versus incident angle $\theta_{i}$ for the case of '-' solution and perpendicular polarization at the wavelengths (a) $\lambda=0.5 \mu \mathrm{m}$, (b) $\lambda=1.0 \mu \mathrm{m}$, (c) $\lambda=1.5 \mu \mathrm{m}$, (d) $\lambda=2.0 \mu \mathrm{m}$. The parameters of the structure are the same as those in Figure 4.1. 
case too, for a range of incident angles $\boldsymbol{\theta}_{i}$ above $\boldsymbol{\theta}_{c}$ the real part of $\bar{Z}_{i n}$ is zero. However, there are ranges of incident angle below $\boldsymbol{\theta}_{c}$ in which $\operatorname{Re}\left[\bar{Z}_{i n}\right]=0$. For example, in Figure 4.3 (b) there exist narrow ranges of incident angles between $\theta_{i}=20^{\circ}$ and $\theta_{i}=30^{\circ}$ and between $\theta_{i}=30^{\circ}$ and $\theta_{i}=40^{\circ}$ where $\bar{Z}_{i n}$ is purely imaginary, resulting in total reflection of incident waves.

The wave impedance for the solutions with a ' + ' sign in (2.27) and (2.37) and for both cases of parallel and perpendicular polarizations were also investigated. The results for these cases possess features very similar to those for the cases corresponding to solutions with a '-' sign presented in Figures 4.1 to 4.4. Much of the discussion on the results in these figures is also applicable to the results for solutions with a ' + ' sign. Thus,

for the sake of brevity, plots of the real and imaginary parts of $\bar{Z}_{i n}$ with the '+' solutions are not given.

In summary, the above results confirm the fact that photonic crystal structures have the ability to totally reflect not only electromagnetic waves incident at angles larger than the critical angle, but also the waves with smaller angle of incidence and even the waves which are normally incident on such structures.

\subsection{Propagation Characteristics of One-Dimensional Planar Photonic Crystal Waveguides}

An important property of guided modes in photonic crystal waveguides is the axial propagation constant $\beta$, which, in addition to describing the z-dependence of the modes through the propagation term $\exp (-\mathrm{j} \beta \mathrm{z})$, is needed in calculating the modal fields, dispersion, and phase and group velocities. It also plays an important role in situations where mode coupling takes place. For one-dimensional planar photonic crystal waveguides, the propagation constant is calculated from the characteristic equations (3.7) and (3.14) when the cladding has infinite number of layers and from (3.56) and (3.57) 
when the number of cladding layers is finite. These equations for given waveguide parameters and a given wavelength become a function of $\beta$ only which can be solved using numerical techniques. Here, we employ the intervathalving method to find the roots of the characteristic equations. The results are presented as plots of normalized propagation constant $\bar{\beta}=\beta / k_{0} \quad\left(k_{0}=2 \pi / \lambda\right.$ is the free-space wave number $)$ versus wavelength $\lambda$ for different modes.

Let us consider, as an example, a one-dimensional photonic crystal waveguide with parameters $a=1 \mu m, d_{1}=1 \mu m, d_{2}=1 \mu m, n_{1}=1.5$, and $n_{2}=1$. The $\bar{\beta}$ values are calculated for a range of wavelengths varying between $0.8 \mu \mathrm{m}$ to $1.8 \mu \mathrm{m}$, in increments of $0.1 \mu \mathrm{m}$. For the infinite-layer cladding, the solutions for $\bar{\beta}$ in the range of $n_{2}<\bar{\beta}<n_{1}$ is calculated from (3.7), while for $\bar{\beta}$ in the range of $0<\bar{\beta}<n_{2}$ it is calculated from (3.14). The roots are obtained with an accuracy in the range of $-10^{-10}$ to $+10^{-10}$. The solutions are obtained for both TM and TE modes. Each group of TE or TM modes can be designated as even or odd. In (3.7) and (3.14), the odd TE or TM modes correspond to $\mathrm{m}=1$, while even TE or TM modes correspond to $\mathrm{m}=0$. The search for solutions is repeated at each wavelength in the above mentioned range to find the first two lower order modes; namely, the first and the second even or odd, TE or TM, modes. We compare the results for the normalized propagation constant with those obtained for a waveguide with the same parameters, but with a finite number of cladding layers. With a sufficiently large number of cladding layers, the waveguide with a finite cladding closely approximates the waveguide with an infinite periodic cladding. This allows us to compare the results for $\bar{\beta}$ obtained from two different approaches, and thereby ascertain their correctness.

For the example waveguide described above, the two results, we obtained by the novel impedance approach and another by the conventional method of imposing boundary conditions, agree very well. In fact, for a sufficiently large number of layers, both solutions of $\bar{\beta}$ become exactly the same for up 5 to 8 decimal points. Figure 4.5 


\section{Finite Vs. Infinite Layers}

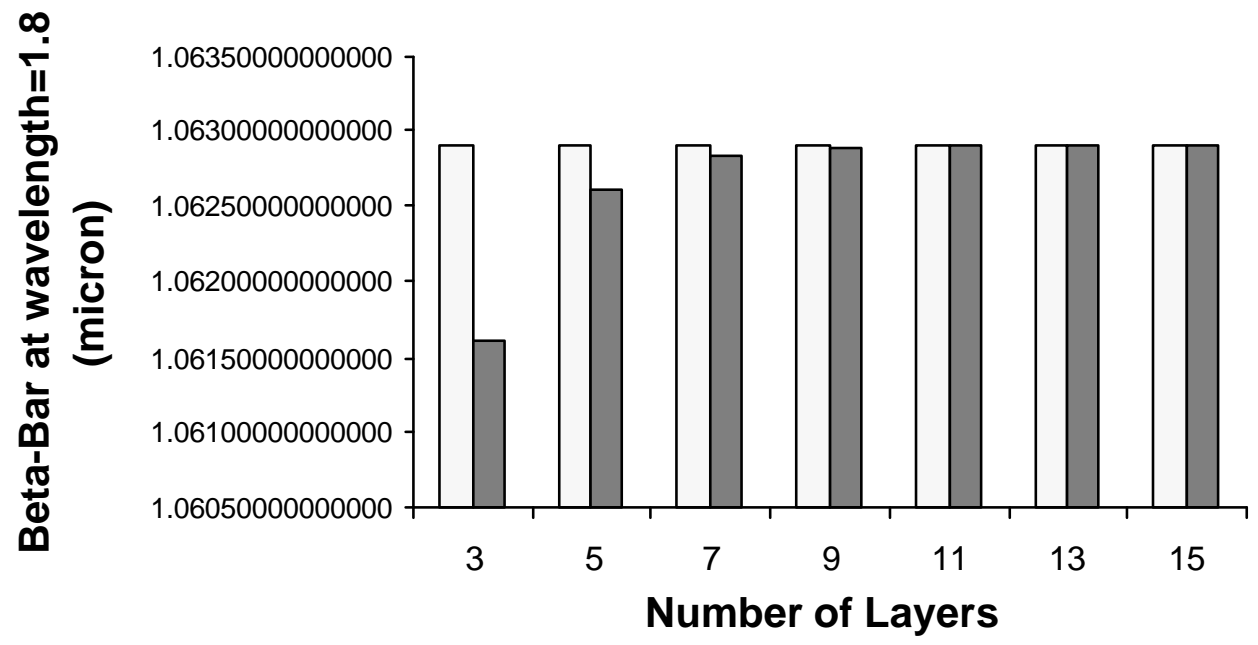

Figure 4.5 Comparison of the normalized propagation constant of $\mathrm{TE}_{2}$ mode at $\lambda=1.8$ $\mu \mathrm{m}$ for waveguides with finite and infinite cladding layers.

compares $\bar{\beta}$ values at $1.8 \mu \mathrm{m}$ wavelength for waveguides with finite and infinite cladding layers. It can be seen, the solution of finite cladding waveguide approaches that of the infinite cladding waveguide as the number of layers is increased.

Next, we examine the propagation characteristics of lower-order modes for the selected example waveguide. Figure 4.6 illustrates variations of the normalized propagation constant versus wavelength for eight modes. For these modes, the normalized propagation constant lies between $n_{2}=1$ and $n_{1}=1.5$; thus guidance is due to the index difference effect. As noted, the normalized propagation constants of all modes decrease with wavelength. At very short wavelengths, $\bar{\beta}$ is slightly below the index $n_{1}$. As wavele ngth increases, $\bar{\beta}$ of each mode at some point becomes equal to $n_{2}$. In conven- 


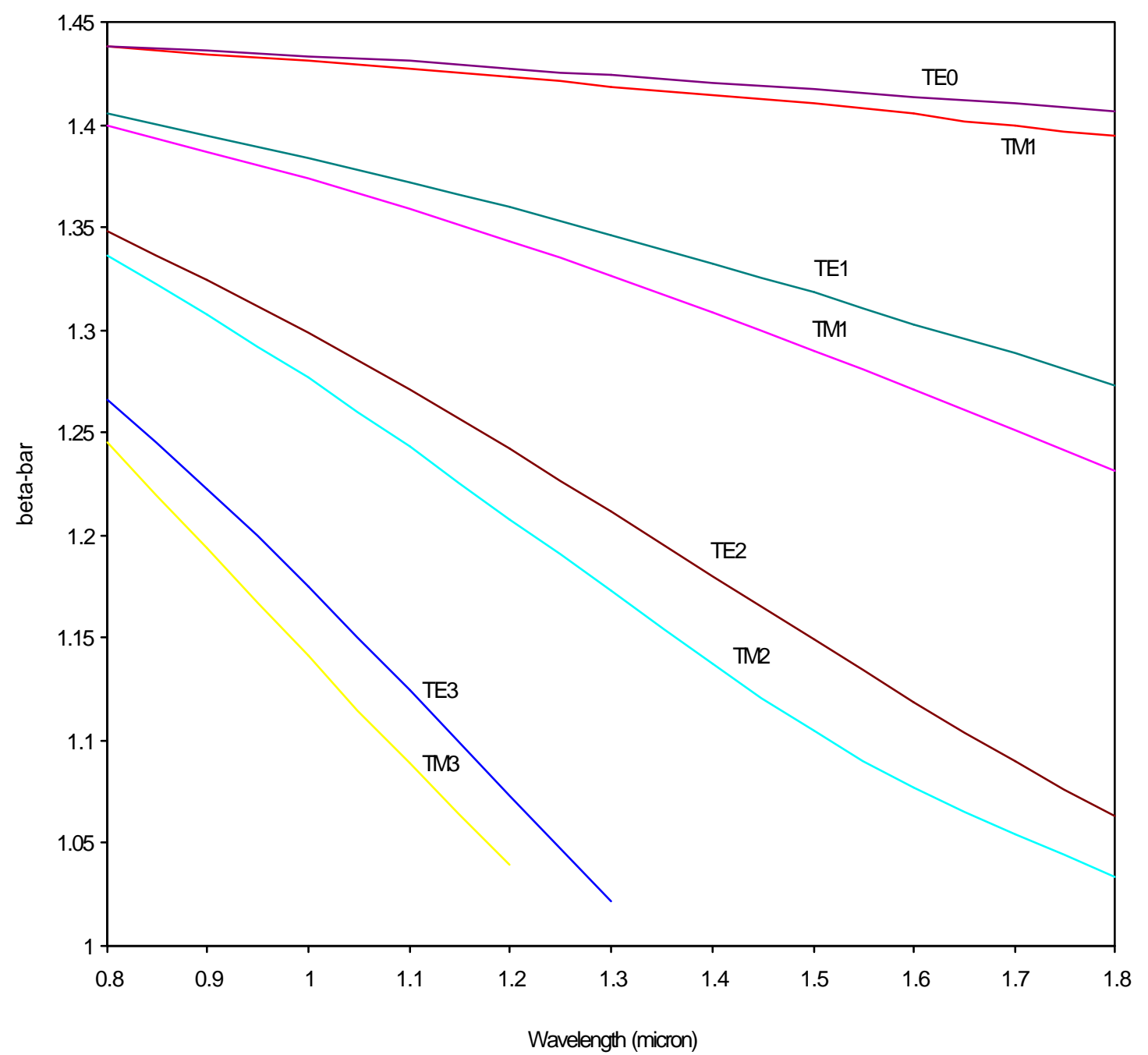

Figure 4.6 Variations of normalized propagation constant versus wavelength for several lower-order modes in a planar photonic crystal waveguide with parameters $a=1 \mu m, d_{1}=1 \mu m, d_{2}=1 \mu m, n_{1}=1.5$, and $n_{2}=1$. 
tional waveguides, $\bar{\beta}=n_{2}$ corresponds to cutoff. In such waveguides, no real solutions for $\bar{\beta}<n_{2}$ exist, and a mode above its cutoff wavelength begins to lose its power due to radiation. In photonic crystal waveguides, however, real solutions for $\bar{\beta}<n_{2}$ do exist. Figure 4.7 illustrate such solutions for the example waveguide under consideration. In fact, the curves for $\mathrm{TE}_{2}$ and $\mathrm{TM}_{2}$ modes in this figure are the continuations of the curves for the corresponding modes in Figure 4.6.

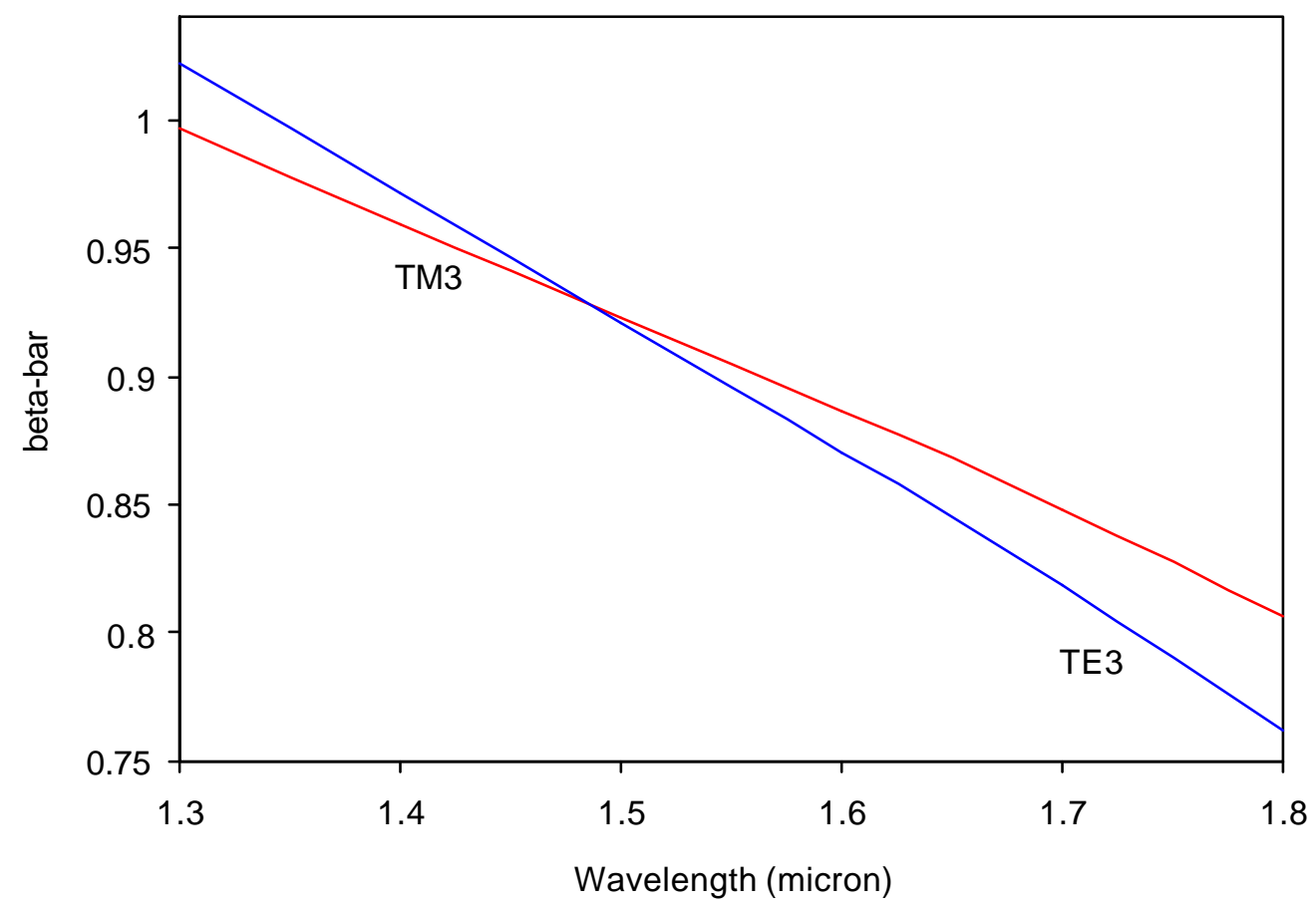

Figure 4.7 Variations of normalized propagation constant versus wavelength for modes supported due to the photonic crystal effect. Waveguide parameters are the same as those in Figure 4.6.

\subsection{Field distributions}

Using the results for normalized propagation constant $\bar{\beta}$ calculated in the previous section, the spatial distribution of field components for several lower-order 
modes of the example waveguide are determined. Field plots for the first three TE modes at the wavelength $\lambda=1.5 \mu \mathrm{m}$ are presented. For the first even TE mode $\left(\mathrm{TE}_{0}\right)$, at this wavelength $\bar{\beta}=1.417627$ is obtained. Using this value in (3.19), (3.27), and (3.28), solutions for the field components $h_{z}, e_{y}$, and $h_{x}$ are obtained. As noted in figure (4.8), $e_{y}$ and $h_{z}$ components, which constitute the tangential components, are continuous everywhere, whereas $h_{x}$, a normal field component, is continuous because the waveguide material is been assumed to be nonmagnetic. All field components decay rapidly in the cladding, essentially becoming zero for $x>2 \mu \mathrm{m}$. Another attribute of the fields for this mode is that the transverse components $e_{y}$ and $h_{x}$ possess only one peak in the central core region.

Field distributions for first odd TE mode $\left(\mathrm{TE}_{1}\right)$, second even TE mode $\left(\mathrm{TE}_{2}\right)$, and the second odd TE mode $\left(\mathrm{TE}_{3}\right)$ are shown in Figures 4.9, 4.10, and 4.11, respectively. It is worth nothing that the transverse components of $\mathrm{TE}_{1}$ mode have two peaks (only half the number of peaks are shown in the figure), while those of the $\mathrm{TE}_{2}$ mode have three peaks in the central core region. Other features of these modes such as rapid decay in the cladding and continuity of $e_{y}$ and $h_{z}$ components are similar to those of the first even TE mode. Furthermore, it should also be noted that the transverse components of even modes $\left(\mathrm{TE}_{0}\right.$ and $\left.\mathrm{TE}_{2}\right)$ exhibit a peak at the center $(x=0)$, while those of the odd modes have a null at the center. 


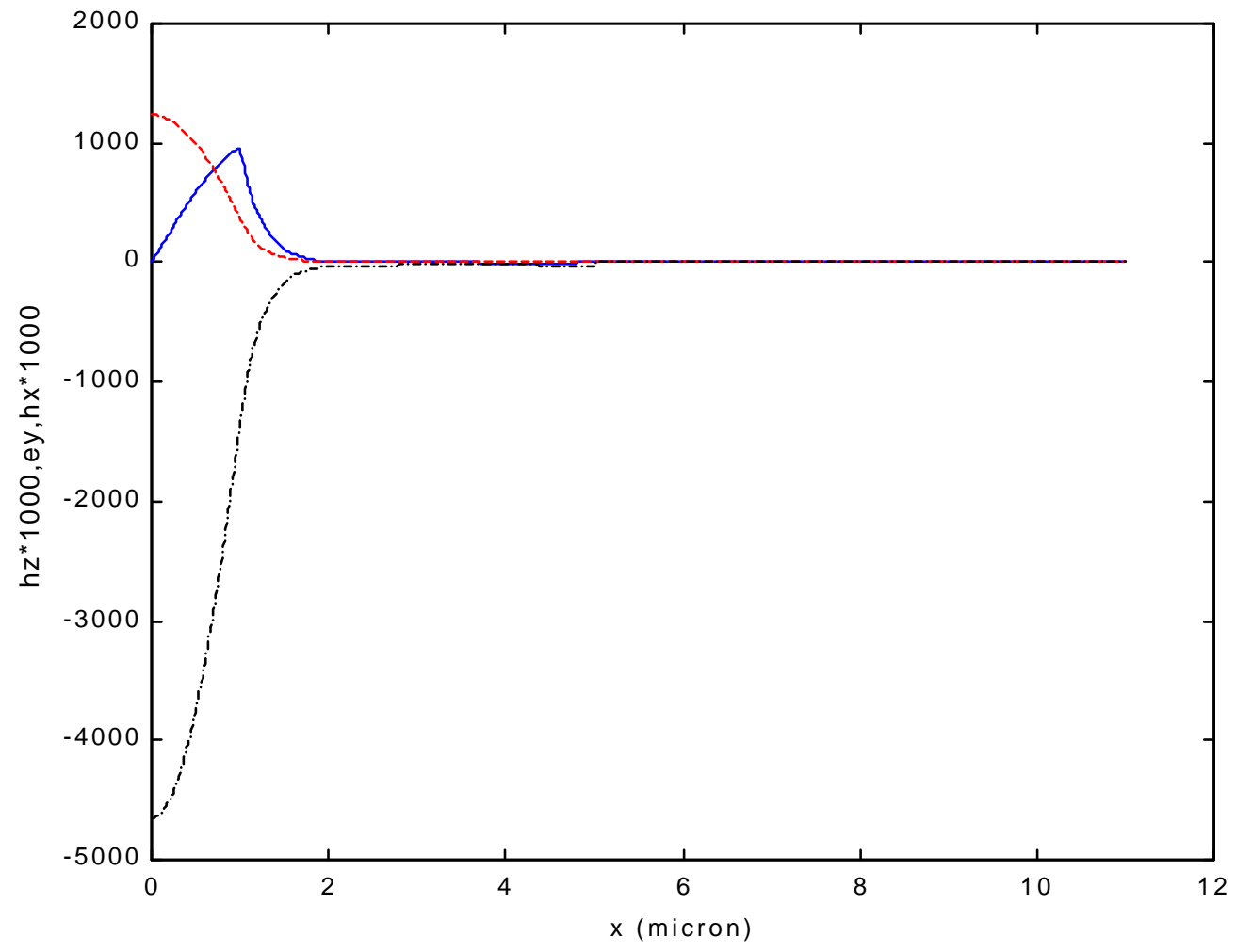

Figure 4.8 Field distributions for the first even TE mode $\left(\mathrm{TE}_{0}\right)$ at $\lambda=1.5 \mu m$. The waveguide parameters are the same as those in Figure 4.6. Solid line for $h_{z}$ multiplied by 1000, dashed line for $e_{y}$, and dashdot line for $h_{x}$ multiplied by 1000 . 


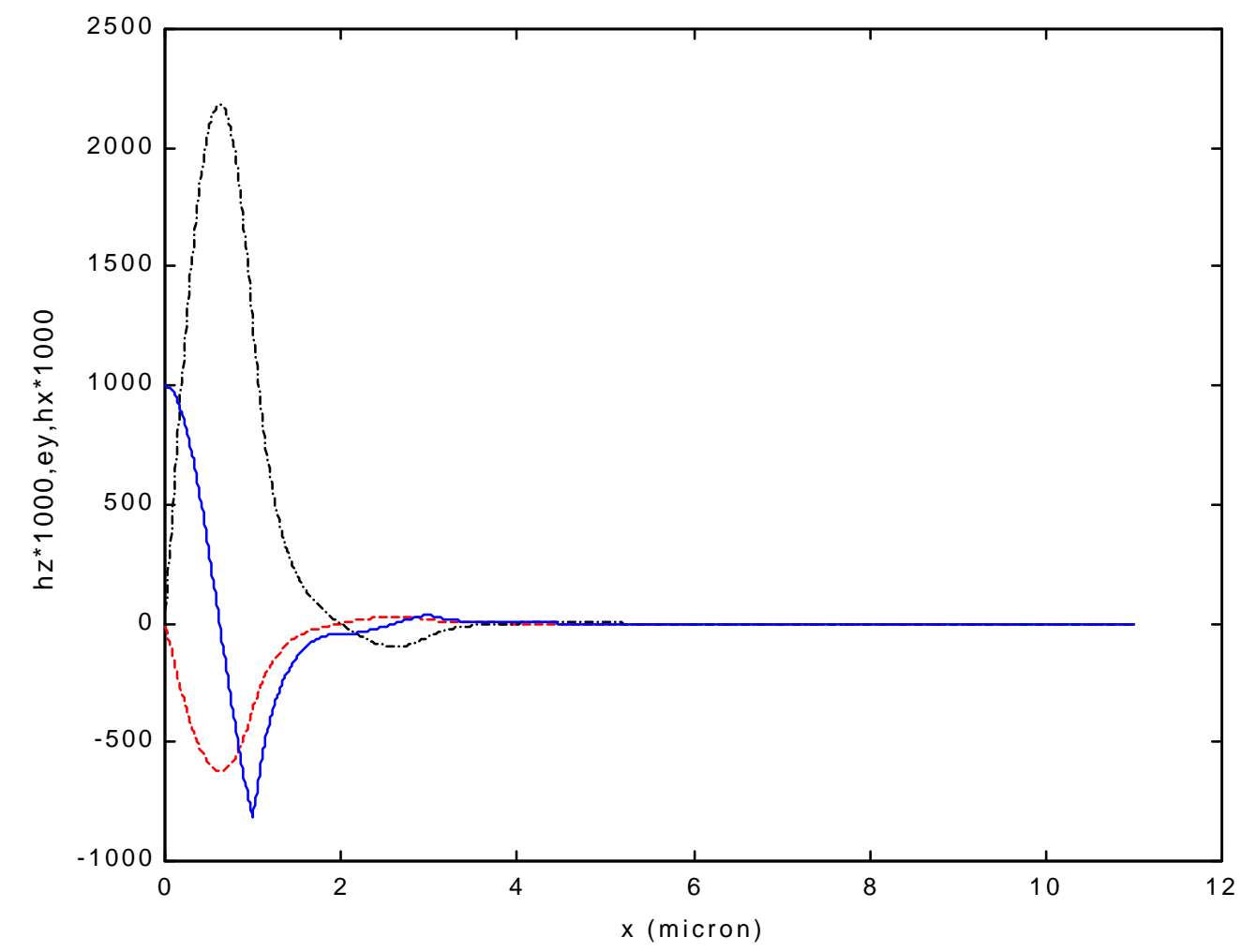

Figure 4.9 Field distributions for the first odd TE mode $\left(\mathrm{TE}_{1}\right)$ at $\lambda=1.5 \mu m$. The waveguide parameters are the same as those in Figure 4.6. Solid line for $h_{z}$ multiplied by 1000, dashed line for $e_{y}$, and dashdot line for $h_{x}$ multiplied by 1000 . 


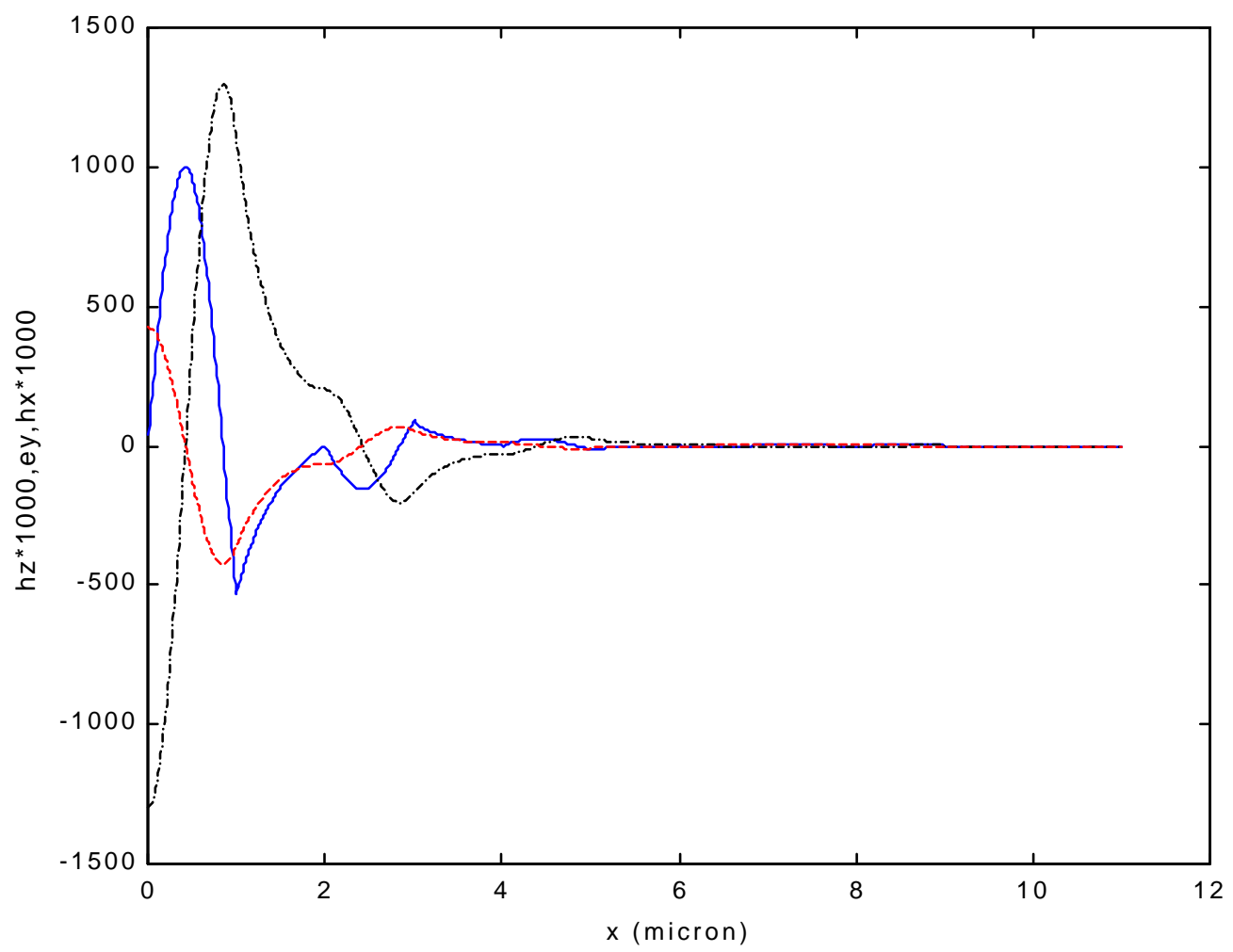

Figure 4.10 Field distributions for the second even TE mode $\left(\mathrm{TE}_{2}\right)$ at $\lambda=1.5 \mu m$. The waveguide parameters are the same as those in Figure 4.6. Solid line for $h_{z}$ multiplied by 1000, dashed line for $e_{y}$, and dashdot line for $h_{x}$ multiplied by 1000 . 


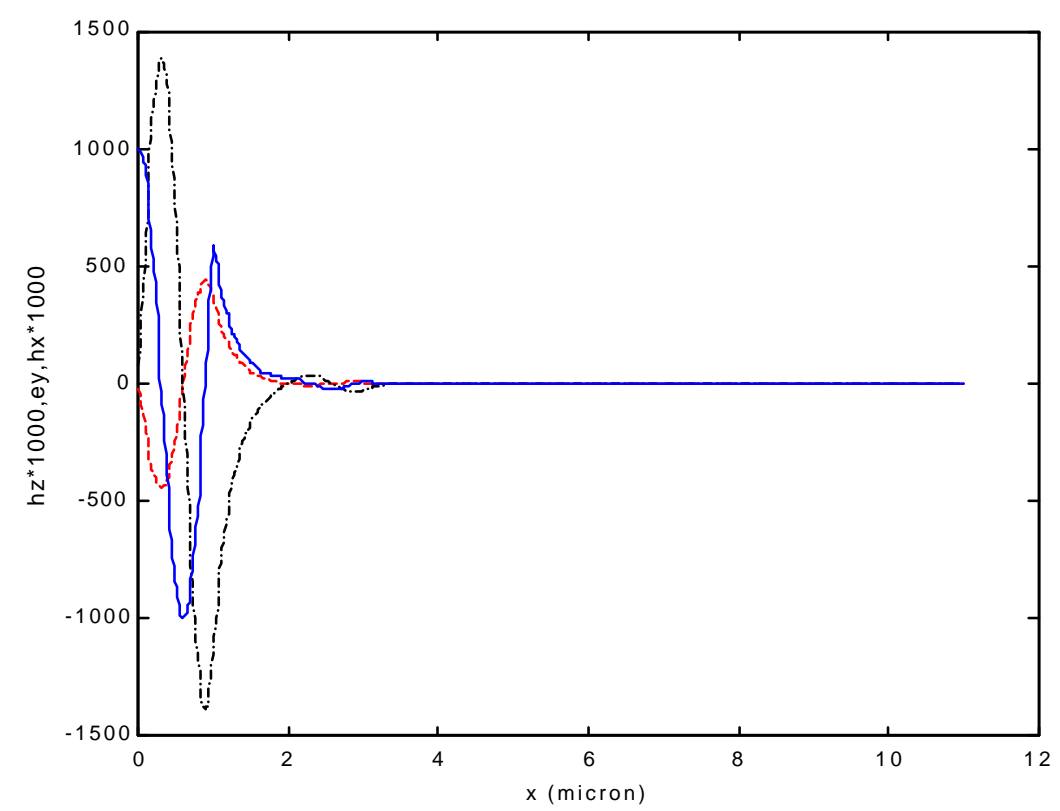

(a)

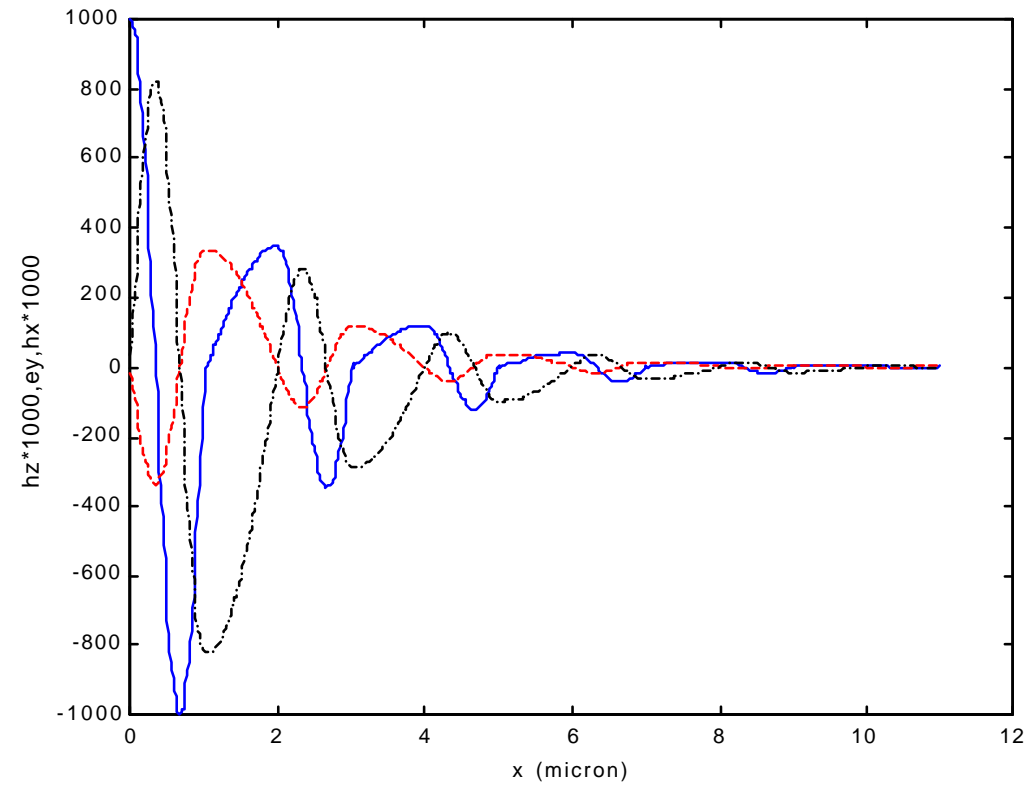

(b)

Figure 4.11 Field distributions for the second odd TE mode $\left(\mathrm{TE}_{3}\right)$ at a) $\lambda=1 \mu \mathrm{m}$ for $\bar{\beta}>\mathrm{n}_{2}$, and b) $\lambda=1.5 \mu \mathrm{m}$ for $\bar{\beta}<\mathrm{n}_{2}$. The waveguide parameters are the same as those in Figure 4.6. Solid line for $h_{z}$ multiplied by 1000, dashed line for $e_{y}$, and dashdot line for $h_{x}$ multiplied by 1000 . 


\section{Chapter 5. Conclusions and Suggestions for Further Research}

\subsection{Conclusions}

In this thesis a novel idea of analyzing the propagating modes in one-dimensional planar photonic crystal waveguides with infinite periodic cladding have been presented. By finding the exact reflection coefficient of a plane wave incident at an arbitrary angle on a semi-infinite periodic dielectric structure, the propagation properties of guided modes in the one-dimensional planar photonic crystal waveguide were studied. The results of the new approach to the results of the boundary-condition approach applied to a waveguide with a finite number of layers were compared, only to conclude that they both match very closely. Thus, the validity of the results were verified and those results which were only unique to photonic crystal structures were clearly distinguished.

It has shown that the propagation constant values obtained from two totally different approaches were essentially the same for a selected waveguide. However, for an infinite photonic crystal waveguide, it was shown that additional solutions exist below the critical angle, where by some modes are able to propagate. This was only realized by deriving an exact formulation for finding the characteristic equation. The theoretical investigation of one-dimensional planar photonic crystal waveguides has enabled finding exactly the propagation properties of guided modes through a more convenient method without any approximations.

\subsection{Suggestions for Further Research}

The main focus of this investigation was to find an analytical solution for the characteristic equations from which the guided modes could be obtained. Based on past 
research and the example cases that have been presented in the previous chapter, there are clearly many factors such as the contrast between refractive indices of the periodic layers, their width, and the position of the defects, which affect the guidance of the modes in the photonic crystal waveguides. However, it was not intended to study any specific property of those waveguides in any detail. Rather, a convenient method was presented, through which the many properties of one-dimensional planar photonic crystals can be studied in more detail. Indeed, the study of photonic crystals is an ongoing research; here yet another tool has been given, from which our understanding of all photonic crystal structures can be enhanced. Additional research may use this new tool to achieve the goal of using these structures at their fullest capability. 


\section{References}

[1] Telecom Report, "Photonic crystals may make all-optical lightwave networks possible," http://www.opticsreport.com/, November 01, 2001.

[2] E. Yablonovitch, "Inhibited spontaneous emission in solid-state physics and electronics," Phys. Review. Letters, Vol. 58, No. 2, p. 2059, 1987.

[3] R. Moussa, "Large photonic band gaps in one-dimensional multiplayer structures," Journal of Physics and Chemistry of Solids, Vol. 63, pp. 725-732, 2002.

[4] J.C. Knight, T.A. Birks, B. Mangan and P.St.J. Russell, "Photonic Crystal Fibers: New Solutions in Fiber Optics," Optics and Photonics News, Vol. 13, March 2002.

[5] R. F. Cregan, B.J. Mangan, J. C. Knight, T. A. Birks, P.St.J. Russell, P.J. Roberts and D.C. Allan, "Single-Mode Photonic Band Gap Guidance of Light in Air," Science, Vol. 285, pp.1537-1539, Sept. 1999.

[6] J. C. Knight, T. A. Birks, P. St. J. Russell, and J. G. Rarity, "Bragg scattering from an obliquely illuminated photonic crystal fiber," Applied Optics, Vol. 37, pp. 449-452, Jan. 1998.

[7] Y. Fink, D. J. Ripin, et al., "Guiding optical light in air using an all-dielectric structure," Journal of Lightwave Technology, Vol. 17, pp. 2039-2041, Nov. 1999.

[8] B. J. Mangan, J. C. Knight, et al., "Experimental study of duatcore photonic crystal fibre," Electronics Letters, Vol. 36, pp. 1358-1359, Aug. 2000.

[9] T. A. Birks, J. C. Knight, and P. St. J. Russell, "Endlessly single-mode photonic crystal fiber,” Optics Letters, Vol. 22, pp. 961-963, July 1997.

[10] Y. Fink, A.M. Urbas, M.G. Bawendi, J.D. Joannopoulos, E.L. Thomas, "Block copolymers as photonic bandgap materials," Journal of Lightwave Technology, Vol. 17, Issue 11, pp. 1963 -1969, Nov. 1999.

[11] Y. Pang; B. Gao, "A new sandwich structure of photonic bandgap," Microwave Symposium Digest, 2002 IEEE MTT-S International, Vol. 2, 2-7, pp. $1157-1160$, June 2002.

[12] B. E. Nelson, M. Gerken, D. A. B. Miller, R. Piestun, C. Lin, Jr. J. S. Harris, "Using of a dielectric stack as a one-dimensional photonic crystal for wavelength demultiplexing by beam shifting," Optics Letters, Vol. 25, No. 20, October 2000. 
[13] J. C. Knight, J. Broeng, T. A. Birks and P. St. J. Russell, "Photonic Band Gap Guidance in Optical Fibers," Science, Vol. 282, pp. 1476-1478, Nov. 1998.

[14] R. Ziolkowski and M. Tanaka, "FDTD analysis of PBG waveguides, power splitters and switches." Optical and Quantum Electronics, Vol. 31, p. 843, Oct. 1999.

[15] E. Centeno, D. Felbacq, "Guiding waves with photonic crystals," Optics Communications, Vol. 160, pp. 57-60, 1999.

[16] C. S. Kee, J. E. Kim, H.Y. Park, H. Lim, "Roles of wave impedance and refractive index in photonic crystals with magnetic and dielectric properties," Microwave Theory and Techniques, IEEE Transactions on, Vol. 47, Issue 11, pp. 2148 -2150, Nov. 1999.

[17] C. S. Kee, J. E. Kim, H. Y. Park, S. J. Kim, H. C. Song, Y. S. Kwon, N. H. Myung, S. Y. Shin, and H. Lim, "Essential parameter in the formation of photonic band gaps," Phys. Rev. E, Vol. 59, No. 4, pp. 4695-4698, Apr. 1999.

[18] M. Lonèar, J. Vuèkoviæa and A. Scherer, "Modal analysis of waveguides based on a triangular photonic crystal lattice," Electrical Engineering Department, California Institute of Technology, July 2000.

[19] T. Søndergaard, A. Bjarklev, M. Kristensen, J. Erland and J. Broeng, "Designing finite-height two-dimensional photonic crystal waveguides," Appl. Phys. Lett., Vol. 77, p. 785, Aug. 2000.

[20] M. E. Zoorob, G. J. Parker, M. C. Netti, J. J. Baumberg, S. Cox, H. Kemhadjian, "Experimental investigation of photonic crystal waveguide devices and linedefect waveguide bends," Materials Science and Engineering B, Vol. 74, pp.1724, 2000.

[21] S. Assefa, A. A. Erchak, D. J. Ripin, S. G. Johnson, M. Mondol, G. S. Petrich, E. P. Ippen, J. D. Joannopoulos, H. I. Smith and L. A. Kolodziejski, "Guiding Light Through Sharp Bends Using Two Dimensional Photonic Crystals," Sponsorship NSF.

[22] L.M. Brekhovskikh, Waves in Layered Media, Ch. 1, Sec. 5, Academic Press, 1960.

[23] J. E. Midwinter, Optical Fibers for Transmission, Wiley, New York, p. 240, 1979.

[24] A. Safaai-Jazi, Lecture Notes on Optical Waveguides, ECE Dept., Virginia Polytechnic Institute and State University, 2001. 
[25] J. A. Kong, "Electromagnetic Wave Theory,” Wiley, New York, p. 150, 1985. 


\section{Vita}

Soheilla Mirlohi was born in Tehran, Iran, in March 1968. She graduated from Blacksburg high school and went on to get her Bachelor of Science degree in Computer Science at VA Tech. She later continued her education in the graduate program in electrical engineering. Since 1993, she has been working for Motion Control Systems, Inc. in New River, Virginia as a Software Engineer. 